\title{
Proteomic analysis of the bone marrow microenvironment in extramedullary multiple myeloma patients
}

\author{
Jana GREGOROVA ${ }^{1}$, Petra VYCHYTILOVA-FALTEJSKOVA ${ }^{1,2}$, Tereza KRAMAROVA ${ }^{1}$, Zdenka KNECHTOVA ${ }^{3}$, Martina ALMASI ${ }^{4}$, Martin STORK ${ }^{3}$, \\ Ludek POUR ${ }^{3}$, Jiri KOHOUTEK ${ }^{5}$, Sabina SEVCIKOVA ${ }^{1, \star}$ \\ ${ }^{1}$ Babak Myeloma Group, Department of Pathophysiology, Faculty of Medicine, Masaryk University, Brno, Czech Republic; ${ }^{2}$ Centre for Molecular \\ Medicine, Central European Institute of Technology (CEITEC), Masaryk University, Brno, Czech Republic; ${ }^{3}$ Department of Internal Medicine, \\ Hematology and Oncology, University Hospital Brno, Brno, Czech Republic; ${ }^{4}$ Department of Clinical Hematology, University Hospital Brno, \\ Brno, Czech Republic; ${ }^{5}$ Section of Genetics and Molecular Biology, Department of Experimental Biology, Faculty of Science, Masaryk University, \\ Brno, Czech Republic
}

*Correspondence: sevcik@med.muni.cz

Received May 27, 2021 / Accepted September 7, 2021

\begin{abstract}
Multiple myeloma (MM) is a heterogeneous hematological malignancy characterized by the uncontrolled clonal proliferation of bone marrow (BM) plasma cells. The poor prognosis of patients is associated with the presence of extramedullary disease (EMD). Previously, different mechanisms involved in the colonization of BM niches by MM cells and their escape during EMD have been described. Thus, we aimed to investigate the expression of selected cytokines in the BM plasma of MM patients as well as EMD patients to reveal novel molecules involved in EMD pathogenesis. Expression of 120 different cytokines was measured in BM plasma of 13 MM and 11 EMD patients using Proteome Profiler Antibody Arrays. The correlation between statistically significant cytokines and clinicopathological parameters of patients was determined using the Spearman correlation analysis. Finally, protein-protein interactions were analyzed, and GO and KEGG pathways enrichment analysis was performed. In total, 27 cytokines were found to be differently expressed between MM and EMD patients. After the Benjamini-Hochberg correction for multiple testing, the statistical significance of two cytokines downregulated in EMD (EGF, BDNF) and six cytokines upregulated in EMD (NAP-2, ADIPOQ, CRP, MIG, BAFF, and THBS1) was maintained. Correlation analysis proved a significant association between the expression of these molecules and selected clinical-pathological features of MM/EMD patients. Protein association network analysis revealed important protein-protein interactions between THBS1/EGF, MIG/NAP-2, THBS1/NAP-2, EGF/NAP-2, and ADIPOQ/CRP. Finally, identified cytokines were proved to be significantly involved in focal adhesion, PI3K/AKT, and MAPK signaling pathways, and regulation of cell development, localization, proliferation, migration, differentiation, immune system processes, and stress response. Obtained results confirm the key function of the BM microenvironment in the pathogenesis of MM and indicate the essential role of numerous cytokines in disease progression and EMD development. However, the exact mechanisms need to be further clarified.
\end{abstract}

Key words: multiple myeloma, extramedullary disease, cytokine, bone marrow microenvironment, protein association network analysis

Multiple myeloma (MM) is the second most common hematological malignancy [1] characterized by clonal proliferation of plasma cells (PCs) in the bone marrow (BM), presence of monoclonal immunoglobulin in serum and/ or urine, and CRAB (hypercalcemia, renal failure, anemia, bone lesions) features [2]. In recent years, the survival of MM patients has dramatically improved with the introduction of new drugs, including proteasome inhibitors, monoclonal antibodies, immunomodulatory drugs, and CAR-T cells [3-6]. However, MM still remains an incurable disease, and poor prognosis of patients is commonly associated with an aggressive and mostly resistant entity called extramedullary disease (EMD) [7], which may be found at the time of $\mathrm{MM}$ diagnosis (primary EMD) or during the relapse (secondary EMD). In addition, EMD may be divided into two other groups: bone-related EMD (EMD-B) extending directly from osteolytic bone lesions and soft-tissue EMD (EMD-S) resulting from PC infiltration into soft tissues with no relationship to the bone [8]. While EMD-B cells are still partially dependent on the BM microenvironment, EMD-S cells commonly exhibit immature, plasmablastic morphology and carry different biological characteristics [9] resulting in 
the worse clinical outcome of EMD-S patients compared to those with EMD-B $[10,11]$. Finally, plasma cell leukemia (PCL) typical for its rapid progression, drug resistance, and poor survival is sometimes considered an extreme variant of aggressive EMD [12].

Currently, the "seed and soil" hypothesis postulating that tumor cells grow preferentially in the selective microenvironment $[13,14]$ is well accepted in the case of MM and EMD. The BM harbors several types of cells, such as osteoblasts, osteoclasts, fibroblasts, endothelial cells, adipocytes, or cells of the immune system as well as non-cellular components including extracellular matrix (ECM) proteins (laminin, fibronectin, collagen, thrombospondin, proteoglycans) and soluble factors (cytokines, growth factors, soluble isoforms of cell adhesion molecules), which support the growth and development of hematopoietic stem cells [15].

Recently, different trafficking events associated with colonization of BM niches by MM cells and their escape from BM during the extramedullary stages of the disease have been described [16]. The most important molecule mediating homing of both normal and malignant PCs into the $\mathrm{BM}$ is the chemokine receptor CXCR4 interacting with stromal cell-derived factor 1 (SDF-1, CXCL12 chemokine), which is highly expressed in the $\mathrm{BM}$ microenvironment [17]. In addition, this interaction upregulates the expression of the $\alpha 4 \beta 1$ integrin on the surface of MM cells allowing its binding with vascular cell adhesion protein-1 (VCAM-1, CD106) expressed on the BM microvasculature [18]. Importantly, decreased levels of CXCR4 were observed in patients with enhanced expression of macrophage migration inhibitory factor (MIF) [19] as well as in patients treated with bortezomib and were associated with an unwanted clinical response comprising of $\mathrm{MM}$ cells migration from $\mathrm{BM}$ and development of EMD [20].

Upon lodging of MM cells into BM, the niche phenotype is significantly altered by remodeling adhesion receptormediated cell-cell contracts as well as through the release of different cytokines, growth factors, exosomes, or non-coding RNAs, which results in enhanced proliferation and migration of MM cells, reduced apoptosis, and drug resistance $[21,22]$. To date, several soluble mediators were found to be crucial for disease development and progression, including interleukin (IL)-6, IL-8, B-cell activating factor (BAFF), a proliferation-inducing ligand (APRIL), colony stimulating factor-1 (CSF-1), vascular endothelial growth factor (VEGF), or fibroblast growth factor-2 (FGF-2) [23]. In addition, it has been proposed that MM cells contribute to bone resorption by stimulating the expression of receptor activator of nuclear factor kappa-B ligand (RANKL) in BM stromal cells (BMSCs) and by stimulating the production of pro-osteoclastogenic cytokines such as IL-6, IL- $1 \alpha / \beta$, or tumor necrosis factor alpha/beta $(\mathrm{TNF}-\alpha / \beta)[24]$. Besides adhesion molecules, additional chemotactic signals are necessary to enhance the selectivity of MM migration. It was repeatedly proven that MM cells express functional chemokine recep- tors, including CCR1, CCR2, CCR5, CXCR3, and CXCR4 $[17,25-28]$ that mediate their migration to corresponding chemokine ligands, such as macrophage inflammatory protein-1a (MIP-1a, CCL3), SDF-1 (CXCL12) [26, 29], the monocyte chemotactic proteins 1-3 (MCPs 1-3, CCL2/7/8) [27], interferon gamma-induced protein 10 (IP10, CXCL10), interferon-inducible T-cell alpha chemoattractant (I-TAC, CXCL11), or monokine induced by gamma interferon (MIG, CXCL9) [30]. Finally, cytokines may be produced not only by MM cells and BMSCs, but also by immune cells, including T-cells, B-cells, natural killers (NKs), neutrophils, or macrophages, thus regulating the immune response. Some of these cytokines, such as IL-1, IL-6, IL-12, IL-16, interferon gamma $(\mathrm{IFN}-\gamma)$, or TNF- $\alpha$ have proinflammatory effects, while others including IL-4, IL-10, IL-11, and TGF- $\beta 1$ exert antiinflammatory activity [31].

As previous reports indicated the crucial role of soluble molecules in the pathogenesis of MM, the aim of this study was to investigate the expression of 120 different cytokines with the potential involvement in MM development and its progression into EMD using high-throughput proteomic analyses of BM plasma samples. Further, the expression profiles of differentially expressed cytokines were correlated with clinical-pathological data of the patients, and their involvement in basic biological processes and signaling pathways was assessed. To the best of our knowledge, no similar study has been previously published.

\section{Patients and methods}

Patients' characteristics. In total, 24 patients (13 MM and 11 EMD patients) diagnosed at the University Hospital Brno, Czech Republic between the years 2014-2017 were involved in the study. Diagnosis of EMD was based on imaging techniques (X-ray, PET-CT, MRI, low-dose CT) according to clinical indication and confirmed by histopathology analysis of biopsied samples. All MM patients' samples were collected at the time of diagnosis prior to any treatment. All patients signed the informed consent form approved by the Ethics committee of the hospital in accordance with the current version of the Helsinki Declaration. Clinical characteristics of patients are summarized in Table 1 .

Measurement of cytokines. Plasma samples were obtained by centrifugation $\left(2,000 \mathrm{~g} / 15 \mathrm{~min} / 20^{\circ} \mathrm{C}\right)$ of heparinized $\mathrm{BM}$ and frozen at $-80^{\circ} \mathrm{C}$ as $0.5 \mathrm{ml}$ aliquots until analysis. In total, 120 different cytokines were analyzed by Proteome Profiler Antibody Arrays-Human XL Cytokine Array Kit (cat. number ARY022B) and Human Chemokine Array Kit (cat. number ARY017) according to the manufacturer's instructions (R\&D Systems, Canada). The developed radiographic films were scanned and processed using the ImageJ (Image Processing and Analysis in Java, NIH) software supplemented with a Protein Array Analyzer.

Statistical analysis. The obtained signal values were exported and further processed in Microsoft Office Excel. 
Table 1. Clinicopathological parameters of multiple myeloma patients and extramedullary disease patients involved in the study.

\begin{tabular}{|c|c|c|c|}
\hline Clinicopathological parameters & & $\begin{array}{l}\text { Multiple myeloma } \\
\text { (MM, \%) }\end{array}$ & $\begin{array}{c}\text { Extramedullary disease } \\
(\mathrm{EMD}, \%)\end{array}$ \\
\hline \multirow{2}{*}{ Sex } & males & $5(38.0)$ & $6(55.0)$ \\
\hline & females & $8(62.0)$ & $5(45.0)$ \\
\hline \multirow[t]{2}{*}{ Age } & median (min-max) & $70(45-81)$ & $68(54-82)$ \\
\hline & $\mathrm{I}$ & $5(38.0)$ & $3(27.0)$ \\
\hline \multirow[t]{3}{*}{ International Staging System (ISS) } & II & $4(31.0)$ & $4(36.5)$ \\
\hline & III & $4(31.0)$ & $4(36.5)$ \\
\hline & $\mathrm{I}$ & $0(0.0)$ & $0(0.0)$ \\
\hline \multirow[t]{2}{*}{ Durie-Salmon stage (DSS) } & II & $2(15.0)$ & $2(18.0)$ \\
\hline & III & $11(85.0)$ & $9(82.0)$ \\
\hline \multirow{2}{*}{ Durie-Salmon substage } & A & $8(62.0)$ & $6(55.0)$ \\
\hline & $\mathrm{B}$ & $5(38.0)$ & $5(45.0)$ \\
\hline \multirow{7}{*}{ M-protein type } & $\operatorname{IgG}$ & $10(77.0)$ & $3(27.0)$ \\
\hline & $\operatorname{IgA}$ & $2(15.0)$ & $6(55.0)$ \\
\hline & $\operatorname{IgM}$ & $0(0.0)$ & $0(0.0)$ \\
\hline & $\operatorname{IgD}$ & $0(0.0)$ & $0(0.0)$ \\
\hline & light chains only & $1(8.0)$ & $2(18.0)$ \\
\hline & non-secretory & $0(0.0)$ & $0(0.0)$ \\
\hline & polyclonal & $0(0.0)$ & $0(0.0)$ \\
\hline \multirow{2}{*}{ Light chain type } & kappa & $8(62.0)$ & $5(45.0)$ \\
\hline & lambda & $5(38.0)$ & $6(55.0)$ \\
\hline Hemoglobin level $\left(\mathrm{g} \times \mathrm{l}^{-1}\right)$ & median (min-max) & $102.00(82.00-141.00)$ & $103.00(83.00-142.00)$ \\
\hline Thrombocyte count $\left(10 \times 10^{9} \mathrm{I}^{-1}\right)$ & median (min-max) & $236.00(119.00-319.00)$ & $153.00(59.80-239.00)$ \\
\hline Calcium total level $\left(\mathrm{mmol} \times \mathrm{l}^{-1}\right)$ & median (min-max) & $2.34(2.05-3.13)$ & $2.53(2.17-3.93)$ \\
\hline Albumin level $\left(\mathrm{g} \times \mathrm{l}^{-1}\right)$ & median (min-max) & $34.00(21.60-46.50)$ & $39.30(24.40-49.20)$ \\
\hline Creatinine level $\left(\mu \mathrm{mol} \times \mathbf{l}^{-1}\right)$ & median (min-max) & $85.00(45.00-913.00)$ & $95.00(59.00-373.00)$ \\
\hline$\beta_{2}-$ microglobulin level $\left(\mathrm{mg} \times \mathbf{l}^{-1}\right)$ & median (min-max) & $4.62(1.96-50.00)$ & $5.72(2.17-21.00)$ \\
\hline Lactate dehydrogenase level ( $\mu$ kat) & median (min-max) & $3.25(1.41-6.65)$ & $4.65(2.27-18.07)$ \\
\hline $\mathrm{C}$-reactive protein level $\left(\mathrm{mg} \times \mathrm{l}^{-1}\right)$ & median (min-max) & $2.30(0.00-129.00)$ & $6.20(1.30-53.10)$ \\
\hline $\mathrm{M}$-protein serum level $\left(\mathrm{g} \times \mathrm{l}^{-1}\right)$ & median $(\min -\max )$ & $26.00(0.00-62.20)$ & $25.75(3.50-56.00)$ \\
\hline Plasma cells count in bone marrow $(\%)$ & median (min-max) & $19.90(1.00-76.00)$ & $1.80(0.00-45.60)$ \\
\hline
\end{tabular}

The values of poor-quality spots were excluded from the subsequent analyses as part of the image quality control. Further, the averaged background signal of individual images was determined using a signal from a clear area of the particular array and subtracted from the obtained values. The values were then converted to percentages relative to the arithmetic mean of the values of the chip's positive controls. Normalization of values between frames was performed by quantile normalization. The final values were obtained by arithmetic averaging the values from duplicate chip spots. The statistical significance of differences in continuous variables between MM and EMD patients was analyzed using the exact nonparametric Mann-Whitney $U$ test for tied and non-tied data sets [32]. The p-values were subsequently evaluated using the Benjamini-Hochberg correction for multiple testing. The correlation between statistically significant cytokines and clinical-pathological parameters was determined using the Spearman correlation analysis. The results of all analyzes were considered statistically significant at the significance level of $p<0.05$. Statistical analysis was performed in GraphPad Prism 6 (GraphPad Software, USA).

Protein association network analysis. Differentially expressed cytokines were analyzed for protein-protein interactions using Search Tool for the Retrieval of Interacting Genes/Proteins (STRING) v11.0 (https://string-db.org/) and visualized with Cytoscape 3.8.2 software [33]. Subsequently, gene ontology (GO) and Kyoto Encyclopedia of Genes and Genomes (KEGG) pathways enrichment analysis was performed in order to analyze the involvement of selected cytokines in biological processes and signaling pathways [34].

\section{Results}

Different cytokine expression in EMD patients compared to MM patients. In order to identify molecules responsible for $\mathrm{MM}$ progression and associated with EMD, the expression levels of 120 different cytokines were 
measured in BM plasma of $13 \mathrm{MM}$ patients and $11 \mathrm{EMD}$ patients using Proteome Profiler Antibody Arrays. In total, 27 cytokines were found to be differently expressed between these two groups of patients $(\mathrm{p}<0.05)$, including epidermal growth factor (EGF), neutrophil activating peptide 2 (NAP-2), adiponectin (ADIPOQ), C-reactive protein (CRP), brain-derived neurotrophic factor (BDNF), MIG (CXCL9), BAFF (TNFSF13B-TNF superfamily member 13b), thrombospondin 1 (THBS1), IL-8 (CXCL8), platelet derived growth factors $\mathrm{AA} / \mathrm{BB} / \mathrm{AB}$ (PDGF-AA/ $\mathrm{BB} / \mathrm{AB}$ ), myeloperoxidase (MPO), platelet factor 4 (PF4; CXCL4), CCL-28, I-TAC (CXCL11), IL-19, epithelial cellderived neutrophil-activating peptide-78 (ENA-78; CXCL5), macrophage-derived chemokine (MDC; CCL22), VCAM-1 (CD106), vitamin D binding protein (DBP), apolipoprotein A1 (APOA1), regulated upon activation, normal T-cell expressed, and secreted (RANTES; CCL5), T-cell immunoglobulin and mucin domains-containing protein 1 (TIM-1), growth differentiation factor 15 (GDF-15), CXCL16, vascular endothelial growth factor co-regulated chemokine-1 (VCC-1; CXCL17), and urokinase plasminogen activator surface receptor (uPAR; PLAUR) (Table 2). The expression of 13 of these cytokines was significantly downregulated in EMD, while the levels of 14 cytokines were found to be significantly upregulated in EMD compared to MM patients.

After the Benjamini-Hochberg correction for multiple testing, statistical significance of two downregulated cytokines (EGF, BDNF) and six upregulated cytokines (NAP-2, ADIPOQ, CRP, MIG, BAFF, and THBS1) was maintained (Figures $1 \mathrm{~A}-1 \mathrm{H}$; Table 2). Interestingly, the positive correlation between the expression of ADIPOQ/ THBS1 ( $\mathrm{r}=0.674, \mathrm{p}=0.012)$ and BDNF/EGF $(\mathrm{r}=0.589$, $\mathrm{p}=0.034$ ) was observed in $\mathrm{MM}$ patients, while negative correlation between the expression of BAFF/CRP $(r=-0.615$, $\mathrm{p}=0.044), \mathrm{ADIPOQ} / \mathrm{NAP}-2(\mathrm{r}=-0.612, \mathrm{p}=0.045)$, and BDNF/ NAP-2 $(r=-0.671, p=0.024)$ and positive correlation between the levels of BAFF/THBS1 ( $r=0.642, p=0.033)$ was detected in EMD patients.

Correlation of clinicopathological parameters with the levels of differentially expressed cytokines. The association between clinicopathological parameters of MM/EMD patients and eight differentially expressed cytokines was assessed using Spearman correlation analysis. In MM patients, negative correlation between ADIPOQ/lactate dehydrogenase $(\mathrm{LDH})(\mathrm{p}=0.031), A D I P O Q /$ serum CRP $(\mathrm{p}=0.002)$, $\mathrm{BAFF} /$ thrombocytes $(\mathrm{p}=0.019), \mathrm{BDNF} / \mathrm{ISS}$ stage $(\mathrm{p}=0.048)$, $\mathrm{BDNF} / \beta_{2}$-microglobulin $(\mathrm{p}=0.016), \quad \mathrm{CRP} /$ age $(\mathrm{p}=0.003)$, CRP/ISS stage $(\mathrm{p}=0.037), \mathrm{CRP} / \mathrm{Ig}$ isotype $(\mathrm{p}=0.020)$, THBS1/ LDH ( $\mathrm{p}=0.004)$, THBS1/serum CRP $(\mathrm{p}=0.003)$, and NAP- $2 /$ thrombocytes $(p=0.046)$ expression was observed, while the levels of BAFF/albumin ( $\mathrm{p}=0.022), \mathrm{CRP} / \mathrm{BMPC}$ infiltration $(\mathrm{p}=0.018), \mathrm{EGF} /$ thrombocytes $(\mathrm{p}=0.001)$, and MIG/ISS stage $(\mathrm{p}=0.013)$ correlated positively (Table 3 ). In EMD patients, significant negative correlation between the expression of $\mathrm{BAFF} / \mathrm{DS}$ stage $(\mathrm{p}=0.023), \mathrm{BAFF} /$ calcium $(\mathrm{p}=0.001), \mathrm{BAFF} /$ serum M-protein $(p=0.020), B D N F / I g$ isotype $(p=0.007)$, and $\mathrm{EGF} /$ serum CRP ( $\mathrm{p}=0.019)$ was found, while the positive correlation between the levels of $\mathrm{CRP} / \mathrm{calcium}(\mathrm{p}=0.018)$ and THBS1/albumin $(\mathrm{p}=0.039)$ was confirmed (Table 4$)$.

Protein association network analysis. Eight differentially expressed cytokines were analyzed for protein-protein interactions using STRING v11.0 and visualized with Cytoscape 3.8.2 software (Figure 2). The strongest association was observed between THBS1 and EGF (combined score 0.972) based on the results of text mining and curated databases. In addition, a high probability of association was found in the case of MIG-NAP-2 (combined score 0.959), NAP-2-THBS1 (combined score 0.935), EGF-NAP-2 (combined score 0.935), and ADIPOQ-CRP (combined score 0.952). Finally, co-expression of MIG and BAFF was detected using the STRING. Subsequently, GO and KEGG pathways enrichment analysis was performed in order to analyze the involvement of selected cytokines in biological processes and signaling pathways. The most enriched pathways included phosphatidylinositol 3-kinase (PI3K)/AKT signaling pathway, focal adhesion, ras-proximate-1 (RAP1), RAS, and mitogenactivated protein kinase (MAPK) signaling pathways (Figure 2). According to the GO classification of biological processes, the majority of eight deregulated cytokines was found to be involved in the regulation of developmental processes, regulation of localization, cell population proliferation, migration, cell differentiation, regulation of immune system processes, and response to stress (Figure 2).

\section{Discussion}

$\mathrm{MM}$ is a B-cell malignancy characterized by monoclonal expansion of PCs in the BM. Recent studies confirmed the critical dependency of MM cells on the BM microenvironment, which consists of ECM, different types of cells, various soluble factors and provides the signals essential for the growth and survival of tumor cells. Importantly, a subclone of MM cells may become independent of the BM microenvironment, infiltrate other organs or circulate freely in the blood resulting in the development of EMD with a very poor prognosis and resistance to therapy [35].

In this study, we analyzed the expression of 120 different cytokines in BM plasma of MM and EMD patients using Proteome Profiler Antibody Arrays with the aim to identify molecules responsible for the escape of MM cells outside the $\mathrm{BM}$ and development of EMD. In total, 13 downregulated and 14 upregulated cytokines were detected in samples of EMD patients compared to MM patients. After the application of Benjamini-Hochberg correction for multiple testing, the statistical significance of two downregulated cytokines (EGF, BDNF) and six upregulated cytokines (NAP-2, ADIPOQ, CRP, MIG, BAFF, and THBS1) was evaluated and their correlation with selected clinical-pathological parameters of patients was confirmed. Based on the results of GO and KEGG pathways enrichment analysis, most of these 
Table 2. Relative levels of eight cytokines differentially expressed between multiple myeloma patients and extramedullary disease patients (evaluated by exact nonparametric Mann-Whitney U-test for tied and non-tied data sets).

\begin{tabular}{|c|c|c|c|c|c|}
\hline Cytokine & $\begin{array}{c}\text { Multiple myeloma (MM) } \\
\text { Median (25-75\%) } \\
\text { Standard deviation } \\
\end{array}$ & $\begin{array}{c}\text { Extramedullary disease (EMD) } \\
\text { Median }(25-75 \%) \\
\text { Standard deviation } \\
\end{array}$ & p-value & $\begin{array}{l}\text { Benjamini-Hochberg } \\
\text { critical value }\end{array}$ & adj. p-value \\
\hline EGF & $\begin{array}{c}0.444(0.304-0.559) \\
0.209\end{array}$ & $\begin{array}{c}0.102(0.063-0.211) \\
0.113\end{array}$ & 0.00016 & 0.00042 & 0.01872 \\
\hline NAP-2 & $\begin{array}{c}0.695(0.639-0.737) \\
0.117\end{array}$ & $\begin{array}{c}0.794(0.751-0.931) \\
0.112\end{array}$ & 0.00043 & 0.00083 & 0.025482 \\
\hline ADIPOQ & $\begin{array}{c}1.030(0.950-1.110) \\
0.108\end{array}$ & $\begin{array}{c}1.220(1.137-1.240) \\
0.075\end{array}$ & 0.00120 & 0.00125 & 0.04796 \\
\hline CRP & $\begin{array}{c}1.529(1.505-1.628) \\
0.081\end{array}$ & $\begin{array}{c}1.676(1.625-1.734) \\
0.058\end{array}$ & 0.00125 & 0.00167 & 0.03735 \\
\hline BDNF & $\begin{array}{c}0.533(0.257-0.721) \\
0.289\end{array}$ & $\begin{array}{c}0.058(0.032-0.121) \\
0.046\end{array}$ & 0.00247 & 0.00208 & 0.05928 \\
\hline MIG & $\begin{array}{c}0.029(0.021-0.040) \\
0.011\end{array}$ & $\begin{array}{c}0.051(0.036-0.099) \\
0.046\end{array}$ & 0.00249 & 0.00250 & 0.04970 \\
\hline BAFF & $\begin{array}{c}0.155(0.089-0.223) \\
0.089\end{array}$ & $\begin{array}{c}0.454(0.159-0.538) \\
0.319\end{array}$ & 0.00261 & 0.00292 & 0.04474 \\
\hline THBS1 & $\begin{array}{c}0.408(0.305-0.605) \\
0.174\end{array}$ & $\begin{array}{c}0.648(0.584-0.786) \\
0.176\end{array}$ & 0.00330 & 0.00333 & 0.04950 \\
\hline IL-8 & $\begin{array}{c}0.039(0.034-0.048) \\
0.008\end{array}$ & $\begin{array}{c}0.052(0.046-0.076) \\
0.020\end{array}$ & 0.00483 & 0.00375 & 0.06436 \\
\hline PDGF-AB/BB & $\begin{array}{c}0.550(0.429-0.695) \\
0.186\end{array}$ & $\begin{array}{c}0.297(0.230-0.498) \\
0.163\end{array}$ & 0.00509 & 0.00417 & 0.06113 \\
\hline MPO & $\begin{array}{c}0.142(0.089-0.185) \\
0.113\end{array}$ & $\begin{array}{c}0.061(0.030-0.103) \\
0.042\end{array}$ & 0.00565 & 0.00458 & 0.06163 \\
\hline PF4 & $\begin{array}{c}1.028(0.917-1.053) \\
0.084\end{array}$ & $\begin{array}{c}0.892(0.830-0.942) \\
0.071\end{array}$ & 0.00594 & 0.00500 & 0.05943 \\
\hline CCL-28 & $\begin{array}{c}0.021(0.010-0.028) \\
0.012\end{array}$ & $\begin{array}{c}0.004(0.003-0.014) \\
0.009\end{array}$ & 0.00749 & 0.00542 & 0.06913 \\
\hline I-TAC & $\begin{array}{c}0.032(0.024-0.040) \\
0.038\end{array}$ & $\begin{array}{c}0.266(0.038-0.494) \\
0.265\end{array}$ & 0.00885 & 0.00583 & 0.07584 \\
\hline IL-19 & $\begin{array}{c}0.036(0.019-0.130) \\
0.072\end{array}$ & $\begin{array}{c}0.012(0.008-0.026) \\
0.040\end{array}$ & 0.01071 & 0.00625 & 0.08568 \\
\hline ENA-78 & $\begin{array}{c}0.213(0.089-0.319) \\
0.138\end{array}$ & $\begin{array}{c}0.063(0.011-0.099) \\
0.064\end{array}$ & 0.01288 & 0.00667 & 0.09660 \\
\hline MDC & $\begin{array}{c}0.148(0.088-0.174) \\
0.074\end{array}$ & $\begin{array}{c}0.062(0.043-0.116) \\
0.048\end{array}$ & 0.01609 & 0.00708 & 0.11358 \\
\hline VCAM-1 & $\begin{array}{c}1.223(1.183-1.257) \\
0.078\end{array}$ & $\begin{array}{c}1.389(1.349-1.419) \\
0.114\end{array}$ & 0.01751 & 0.00750 & 0.11673 \\
\hline DBP & $\begin{array}{c}1.050(0.838-1.129) \\
0.240\end{array}$ & $\begin{array}{c}0.855(0.717-0.904) \\
0.229\end{array}$ & 0.01836 & 0.00792 & 0.11596 \\
\hline APOA1 & $\begin{array}{c}1.255(1.110-1.356) \\
0.229\end{array}$ & $\begin{array}{c}1.124(1.023-1.189) \\
0.247\end{array}$ & 0.02130 & 0.00833 & 0.12780 \\
\hline RANTES & $\begin{array}{c}0.813(0.770-0.870) \\
0.049\end{array}$ & $\begin{array}{c}0.913(0.833-0.984) \\
0.093\end{array}$ & 0.02175 & 0.00875 & 0.12429 \\
\hline TIM-1 & $\begin{array}{c}0.969(0.884-1.170) \\
0.209\end{array}$ & $\begin{array}{c}1.192(1.094-1.273) \\
0.109\end{array}$ & 0.03012 & 0.00917 & 0.16429 \\
\hline GDF-15 & $\begin{array}{c}0.571(0.306-0.983) \\
0.344\end{array}$ & $\begin{array}{c}0.823(0.648-1.196) \\
0.265\end{array}$ & 0.03123 & 0.00958 & 0.16294 \\
\hline CXCL16 & $\begin{array}{c}0.381(0.348-0.423) \\
0.081\end{array}$ & $\begin{array}{c}0.432(0.406-0.515) \\
0.087\end{array}$ & 0.03468 & 0.01000 & 0.17340 \\
\hline PDGF-AA & $\begin{array}{c}0.648(0.443-0.860) \\
0.265\end{array}$ & $\begin{array}{c}0.367(0.326-0.505) \\
0.254\end{array}$ & 0.03520 & 0.01042 & 0.16896 \\
\hline VCC-1 & $\begin{array}{c}0.041(0.029-0.051) \\
0.014\end{array}$ & $\begin{array}{c}0.028(0.025-0.037) \\
0.008\end{array}$ & 0.04095 & 0.01083 & 0.18900 \\
\hline UPAR & $\begin{array}{c}0.202(0.093-0.362) \\
0.322\end{array}$ & $\begin{array}{c}0.355(0.309-0.403) \\
0.076\end{array}$ & 0.04745 & 0.01125 & 0.21089 \\
\hline
\end{tabular}

Abbreviations: EGF-epidermal growth factor; NAP-2-neutrophil activating peptide 2; ADIPOQ-adiponectin; CRP-C-reactive protein; BDNF-brain-derived neurotrophic factor; MIG-monokine induced by gamma interferon; BAFF-B-cell activating factor; THBS1-thrombospondin 1; PDGF-platelet derived growth factors; MPO-myeloperoxidase; PF4-platelet factor 4; I-TAC-interferon-inducible T-cell alpha chemoattractant; ENA-78-epithelial cell-derived neutrophil-activating peptide-78; MDC-macrophage-derived chemokine; VCAM-1-vascular cell adhesion protein-1; DBP-vitamin D binding protein; APOA1-apolipoprotein A1; RANTES-regulated upon activation, normal T-cell expressed, and secreted; TIM-1-T-cell immunoglobulin and mucin domains-containing protein-1; GDF-15-growth differentiation factor 15; VCC-1-vascular endothelial growth factor co-regulated chemokine-1; uPARurokinase plasminogen activator surface receptor 
A

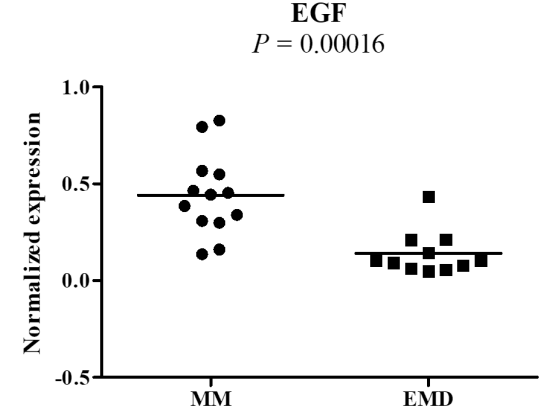

C

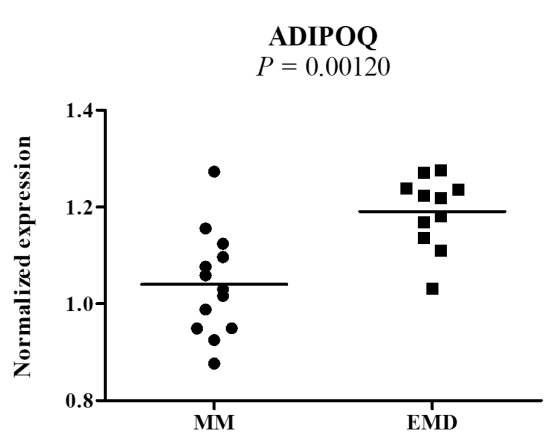

$\mathbf{E}$

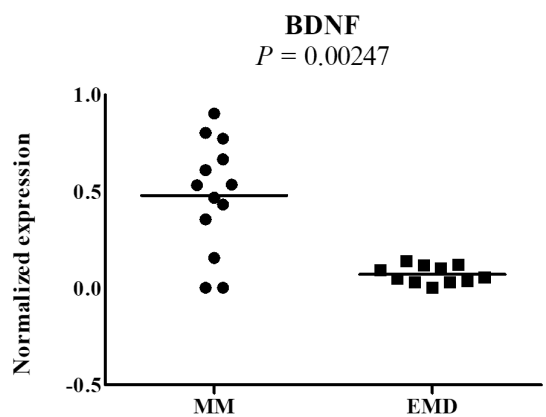

G

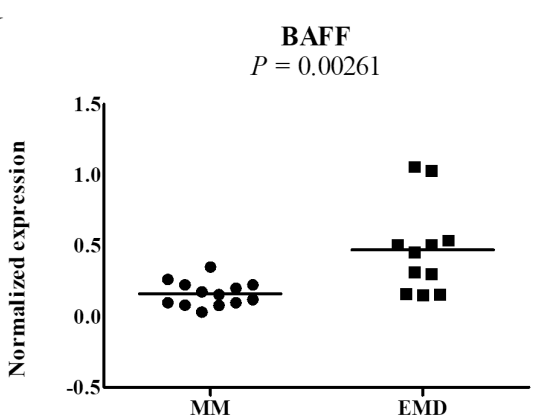

B

NAP-2

$P=0.00043$

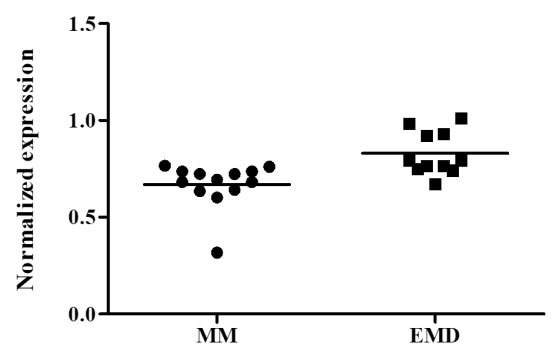

D

CRP

$P=0.00125$

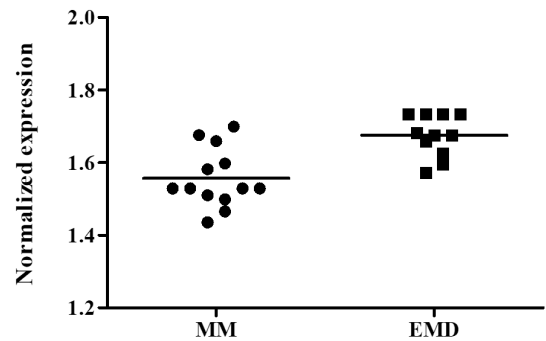

$\mathbf{F}$

MIG

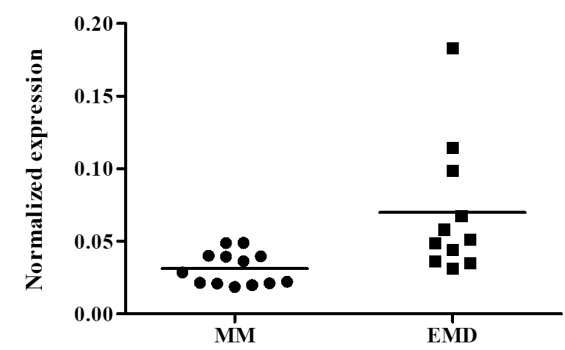

$\mathbf{H}$

THBS1

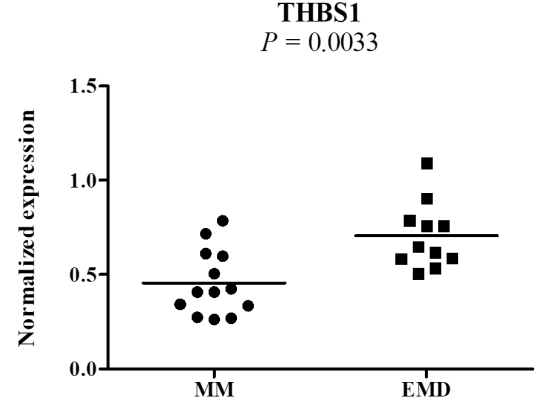

Figure 1. Significantly deregulated cytokines between bone marrow plasma of multiple myeloma patients and patients with extramedullary disease. A) Epidermal growth factor (EGF) is significantly downregulated in the EMD compared to multiple myeloma (MM) $(p=0.00016)$. B) Neutrophil activating peptide 2 (NAP-2) is significantly upregulated in EMD compared to MM ( $\mathrm{p}=\mathbf{0 . 0 0 0 4 3 )}$. C) Adiponectin (ADIPOQ) is significantly upregulated in EMD compared to MM ( $p=0.00120)$. D) C-reactive protein (CRP) is significantly upregulated in EMD compared to $M M(p=0.00125)$. E) Brain-derived neurotrophic factor (BDNF) is significantly downregulated in EMD compared to MM ( $\mathrm{p}=\mathbf{0 . 0 0 2 4 7})$. F) Macrophage migration inhibitory factor (MIG) is significantly upregulated in EMD compared to MM ( $\mathrm{p}=0.00249)$. $\mathrm{G})$ B-cell activating factor (BAFF) is significantly upregulated in EMD compared to MM ( $\mathrm{p}=\mathbf{0 . 0 0 2 4 9 )}$. H) Thrombospondin (THBS1) is significantly upregulated in EMD compared to MM ( $\mathrm{p}=0.0033)$. 
cytokines are involved in focal adhesion and PI3K/AKT, RAP1, RAS, and MAPK signaling pathways as well as in the regulation of localization, development, cell proliferation, migration, differentiation, immune system processes, and response to stress.

So far, there are not many studies investigating the expression profiles of cytokines in BM plasma of MM/EMD patients. Previously, Cao et al. [36] determined the concentrations of 34 different cytokines in the supernatant of ten MM cell lines as well as in the BM plasma and peripheral blood of $10 \mathrm{MM}$ patients, 20 patients with allogeneic stem cell transplantation, and 20 healthy donors. Similar to our study, they confirmed deregulated levels of EGF and MIG in the BM plasma of MM patients. In addition, both proteins together with RANTES and IL-8 that were also confirmed to be significantly deregulated in this study evinced increased levels in MM culture supernatants and elevated concentra- tion of IL-8 was associated with allogeneic transplantation of stem cells.

In another study, the profiles of 27 selected cytokines were assessed in the serum samples of MM patients compared to healthy controls. The authors found significant overexpression of IL-8 and RANTES in MM compared to control samples, while the levels of PDGF-BB were decreased and the concentration levels of IL-8 and PDGF-BB correlated with the disease stage. Further, expression levels of IL-8 together with MIP- $1 \alpha$ and IL- $1 \beta$ enabled prediction of response to bortezomib treatment with the sensitivity of $80.5 \%$ and specificity of 79.3\% [37]. Recently, overexpression of MIG was confirmed in serum samples of MM patients compared to healthy controls or patients with monoclonal gammopathy of undetermined significance (MGUS). Similarly, to our results, increased MIG levels correlated with the advanced ISS stage. In addition, a significant correlation between the

Table 3. Spearman correlation analysis of clinicopathological features with the levels of differentially expressed cytokines in bone marrow plasma of multiple myeloma patients.

\begin{tabular}{|c|c|c|c|c|c|c|c|c|}
\hline $\begin{array}{l}\text { CLINICOPATHOLOGICAL } \\
\text { FEATURE }\end{array}$ & ADIPOQ & BAFF & BDNF & CRP & EGF & MIG & THBS1 & NAP-2 \\
\hline Sex & $\begin{array}{c}-0.042 \\
(\mathrm{p}=0.891)\end{array}$ & $\begin{array}{c}0.233 \\
(\mathrm{p}=0.444)\end{array}$ & $\begin{array}{c}-0.021 \\
(\mathrm{p}=0.945)\end{array}$ & $\begin{array}{c}-0.514 \\
(\mathrm{p}=0.072)\end{array}$ & $\begin{array}{c}0.000 \\
(\mathrm{p}=1.000)\end{array}$ & $\begin{array}{c}0.423 \\
(\mathrm{p}=0.150)\end{array}$ & $\begin{array}{c}-0.127 \\
(\mathrm{p}=0.679)\end{array}$ & $\begin{array}{c}-0.297 \\
(\mathrm{p}=0.325)\end{array}$ \\
\hline Age & $\begin{array}{c}-0.401 \\
(\mathrm{p}=0.174)\end{array}$ & $\begin{array}{c}-0.533 \\
(\mathrm{p}=0.060)\end{array}$ & $\begin{array}{c}0.028 \\
(\mathrm{p}=0.929)\end{array}$ & $\begin{array}{c}-0.752 \\
(p=0.003)\end{array}$ & $\begin{array}{c}0.170 \\
(\mathrm{p}=0.578)\end{array}$ & $\begin{array}{c}0.357 \\
(\mathrm{p}=0.231)\end{array}$ & $\begin{array}{c}-0.179 \\
(\mathrm{p}=0.559)\end{array}$ & $\begin{array}{c}-0.397 \\
(\mathrm{p}=0.180)\end{array}$ \\
\hline ISS stage & $\begin{array}{c}-0.122 \\
(\mathrm{p}=0.691)\end{array}$ & $\begin{array}{c}-0.147 \\
(\mathrm{p}=0.631)\end{array}$ & $\begin{array}{c}-0.557 \\
(p=0.048)\end{array}$ & $\begin{array}{c}-0.581 \\
(p=0.037)\end{array}$ & $\begin{array}{c}-0.425 \\
(\mathrm{p}=0.148)\end{array}$ & $\begin{array}{c}0.667 \\
(\mathrm{p}=0.013)\end{array}$ & $\begin{array}{c}0.039 \\
(\mathrm{p}=0.898)\end{array}$ & $\begin{array}{c}0.069 \\
(\mathrm{p}=0.824)\end{array}$ \\
\hline DS stage & $\begin{array}{c}0.114 \\
(\mathrm{p}=0.711)\end{array}$ & $\begin{array}{c}-0.228 \\
(\mathrm{p}=0.453)\end{array}$ & $\begin{array}{c}-0.342 \\
(\mathrm{p}=0.252)\end{array}$ & $\begin{array}{c}0.260 \\
(\mathrm{p}=0.391)\end{array}$ & $\begin{array}{c}0.057 \\
(\mathrm{p}=0.853)\end{array}$ & $\begin{array}{c}0.171 \\
(\mathrm{p}=0.577)\end{array}$ & $\begin{array}{c}0.257 \\
(\mathrm{p}=0.397)\end{array}$ & $\begin{array}{c}0.229 \\
(p=0.453)\end{array}$ \\
\hline Ig isotype & $\begin{array}{c}-0.259 \\
(\mathrm{p}=0.416)\end{array}$ & $\begin{array}{c}-0.130 \\
(\mathrm{p}=0.688)\end{array}$ & $\begin{array}{c}0.357 \\
(\mathrm{p}=0.255)\end{array}$ & $\begin{array}{c}-0.659 \\
(\mathrm{p}=0.020)\end{array}$ & $\begin{array}{c}0.130 \\
(\mathrm{p}=0.688)\end{array}$ & $\begin{array}{c}0.000 \\
(\mathrm{p}=1.000)\end{array}$ & $\begin{array}{c}-0.130 \\
(\mathrm{p}=0.688)\end{array}$ & $\begin{array}{c}0.033 \\
(\mathrm{p}=0.920)\end{array}$ \\
\hline Type of light-chain & $\begin{array}{c}-0.338 \\
(\mathrm{p}=0.259)\end{array}$ & $\begin{array}{c}-0.444 \\
(\mathrm{p}=0.128)\end{array}$ & $\begin{array}{c}0.000 \\
(\mathrm{p}=1.000)\end{array}$ & $\begin{array}{c}-0.300 \\
(\mathrm{p}=0.319)\end{array}$ & $\begin{array}{c}0.423 \\
(\mathrm{p}=0.150)\end{array}$ & $\begin{array}{c}0.253 \\
(\mathrm{p}=0.403)\end{array}$ & $\begin{array}{c}0.254 \\
(\mathrm{p}=0.403)\end{array}$ & $\begin{array}{c}-0.297 \\
(\mathrm{p}=0.325)\end{array}$ \\
\hline Hemoglobin $\left(\mathrm{g} \times \mathrm{l}^{-1}\right)$ & $\begin{array}{c}-0.187 \\
(\mathrm{p}=0.541)\end{array}$ & $\begin{array}{c}0.150 \\
(\mathrm{p}=0.624)\end{array}$ & $\begin{array}{c}0.384 \\
(p=0.195)\end{array}$ & $\begin{array}{c}0.538 \\
(\mathrm{p}=0.058)\end{array}$ & $\begin{array}{c}0.371 \\
(\mathrm{p}=0.212)\end{array}$ & $\begin{array}{c}-0.360 \\
(\mathrm{p}=0.226)\end{array}$ & $\begin{array}{c}-0.105 \\
(\mathrm{p}=0.734)\end{array}$ & $\begin{array}{c}-0.123 \\
(\mathrm{p}=0.690)\end{array}$ \\
\hline Thrombocytes (amount $\times 10^{9}$ ) & $\begin{array}{c}-0.118 \\
(\mathrm{p}=0.700)\end{array}$ & $\begin{array}{c}-0.639 \\
(\mathrm{p}=0.019)\end{array}$ & $\begin{array}{c}0.532 \\
(\mathrm{p}=0.061)\end{array}$ & $\begin{array}{c}-0.042 \\
(\mathrm{p}=0.892)\end{array}$ & $\begin{array}{c}0.790 \\
(p=0.001)\end{array}$ & $\begin{array}{c}-0.014 \\
(\mathrm{p}=0.964)\end{array}$ & $\begin{array}{c}0.044 \\
(\mathrm{p}=0.886)\end{array}$ & $\begin{array}{c}-0.561 \\
(p=0.046)\end{array}$ \\
\hline Calcium $\left(\mathrm{mmol} \times \mathrm{l}^{-1}\right)$ & $\begin{array}{c}0.088 \\
(\mathrm{p}=0.775)\end{array}$ & $\begin{array}{c}0.492 \\
(\mathrm{p}=0.087)\end{array}$ & $\begin{array}{c}-0.239 \\
(p=0.431)\end{array}$ & $\begin{array}{c}0.504 \\
(\mathrm{p}=0.079)\end{array}$ & $\begin{array}{c}-0.176 \\
(\mathrm{p}=0.566)\end{array}$ & $\begin{array}{c}-0.071 \\
(\mathrm{p}=0.817)\end{array}$ & $\begin{array}{c}0.179 \\
(\mathrm{p}=0.559)\end{array}$ & $\begin{array}{c}0.336 \\
(p=0.262)\end{array}$ \\
\hline Albumin $\left(\mathrm{g} \times \mathrm{l}^{-1}\right)$ & $\begin{array}{c}-0.011 \\
(\mathrm{p}=0.972)\end{array}$ & $\begin{array}{c}0.625 \\
(p=0.022)\end{array}$ & $\begin{array}{c}0.174 \\
(p=0.571)\end{array}$ & $\begin{array}{c}0.462 \\
(\mathrm{p}=0.112)\end{array}$ & $\begin{array}{c}0.014 \\
(p=0.964)\end{array}$ & $\begin{array}{c}-0.294 \\
(\mathrm{p}=0.329)\end{array}$ & $\begin{array}{c}-0.169 \\
(\mathrm{p}=0.580)\end{array}$ & $\begin{array}{c}0.179 \\
(p=0.558)\end{array}$ \\
\hline Creatinine $\left(\mu \mathrm{mol} \times \mathbf{1}^{-1}\right)$ & $\begin{array}{c}-0.093 \\
(\mathrm{p}=0.762)\end{array}$ & $\begin{array}{c}-0.129 \\
(\mathrm{p}=0.674)\end{array}$ & $\begin{array}{c}-0.314 \\
(\mathrm{p}=0.297)\end{array}$ & $\begin{array}{c}-0.454 \\
(\mathrm{p}=0.119)\end{array}$ & $\begin{array}{c}-0.374 \\
(\mathrm{p}=0.209)\end{array}$ & $\begin{array}{c}0.187 \\
(\mathrm{p}=0.541)\end{array}$ & $\begin{array}{c}0.063 \\
(\mathrm{p}=0.837)\end{array}$ & $\begin{array}{c}0.320 \\
(\mathrm{p}=0.287)\end{array}$ \\
\hline $\mathbf{B}_{2}-$ microglobulin $\left(\mathrm{mg} \times \mathbf{l}^{-1}\right)$ & $\begin{array}{c}0.033 \\
(\mathrm{p}=0.915)\end{array}$ & $\begin{array}{c}0.154 \\
(\mathrm{p}=0.615)\end{array}$ & $\begin{array}{c}-0.649 \\
(\mathrm{p}=0.016)\end{array}$ & $\begin{array}{c}-0.423 \\
(\mathrm{p}=0.149)\end{array}$ & $\begin{array}{c}-0.489 \\
(\mathrm{p}=0.090)\end{array}$ & $\begin{array}{c}0.335 \\
(\mathrm{p}=0.263)\end{array}$ & $\begin{array}{c}0.099 \\
(\mathrm{p}=0.748)\end{array}$ & $\begin{array}{c}0.438 \\
(p=0.134)\end{array}$ \\
\hline Lactate dehydrogenase ( $\mu$ kat) & $\begin{array}{c}-0.622 \\
(p=0.031)\end{array}$ & $\begin{array}{c}0.021 \\
(\mathrm{p}=0.948)\end{array}$ & $\begin{array}{c}-0.007 \\
(\mathrm{p}=0.983)\end{array}$ & $\begin{array}{c}-0.488 \\
(\mathrm{p}=0.108)\end{array}$ & $\begin{array}{c}-0.042 \\
(\mathrm{p}=0.897)\end{array}$ & $\begin{array}{c}-0.112 \\
(\mathrm{p}=0.729)\end{array}$ & $\begin{array}{c}-0.767 \\
(p=0.004)\end{array}$ & $\begin{array}{c}-0.035 \\
(\mathrm{p}=0.914)\end{array}$ \\
\hline $\mathrm{C}$-reactive protein $\left(\mathrm{mg} \times \mathrm{l}^{-1}\right)$ & $\begin{array}{c}-0.780 \\
(p=0.002)\end{array}$ & $\begin{array}{c}-0.182 \\
(\mathrm{p}=0.553)\end{array}$ & $\begin{array}{c}0.242 \\
(p=0.426)\end{array}$ & $\begin{array}{c}-0.365 \\
(\mathrm{p}=0.220)\end{array}$ & $\begin{array}{c}-0.060 \\
(\mathrm{p}=0.845)\end{array}$ & $\begin{array}{c}-0.011 \\
(\mathrm{p}=0.972)\end{array}$ & $\begin{array}{c}-0.746 \\
(p=0.003)\end{array}$ & $\begin{array}{c}-0.534 \\
(\mathrm{p}=0.060)\end{array}$ \\
\hline Serum M-protein $\left(\mathrm{g} \times \mathrm{l}^{-1}\right)$ & $\begin{array}{c}0.544 \\
(\mathrm{p}=0.055)\end{array}$ & $\begin{array}{c}0.091 \\
(\mathrm{p}=0.768)\end{array}$ & $\begin{array}{c}-0.402 \\
(\mathrm{p}=0.174)\end{array}$ & $\begin{array}{c}0.100 \\
(\mathrm{p}=0.744)\end{array}$ & $\begin{array}{c}-0.264 \\
(\mathrm{p}=0.384)\end{array}$ & $\begin{array}{c}-0.038 \\
(\mathrm{p}=0.901)\end{array}$ & $\begin{array}{c}0.063 \\
(\mathrm{p}=0.837)\end{array}$ & $\begin{array}{c}0.248 \\
(\mathrm{p}=0.414)\end{array}$ \\
\hline BM infiltration by PCs (\%) & $\begin{array}{c}0.038 \\
(p=0.901)\end{array}$ & $\begin{array}{c}-0.176 \\
(p=0.565)\end{array}$ & $\begin{array}{c}-0.077 \\
(\mathrm{p} 0=0.802)\end{array}$ & $\begin{array}{c}0.641 \\
(p=0.018)\end{array}$ & $\begin{array}{c}0.170 \\
(\mathrm{p}=0.578)\end{array}$ & $\begin{array}{c}-0.198 \\
(\mathrm{p}=0.517)\end{array}$ & $\begin{array}{c}0.083 \\
(p=0.789)\end{array}$ & $\begin{array}{c}0.102 \\
(p=0.740)\end{array}$ \\
\hline
\end{tabular}

Note: significant correlations $(\mathrm{p}<0.05)$ are highlighted in bold

Abbreviations: ISS-International Staging System; DS-Durie Salmon stage; BM-bone marrow; ADIPOQ-adiponectin; BAFF-B-cell activating factor; BDNFbrain-derived neurotrophic factor; CRP-C-reactive protein; EGF-epidermal growth factor; MIG-monokine induced by gamma interferon; THBS1-thrombospondin 1; NAP-2-neutrophil activating peptide 2 
Table 4: Spearman correlation analysis of clinicopathological features with the levels of differentially expressed cytokines in bone marrow plasma of extramedullary disease patients.

\begin{tabular}{|c|c|c|c|c|c|c|c|c|}
\hline $\begin{array}{l}\text { CLINICOPATHOLOGICAL } \\
\text { FEATURE }\end{array}$ & ADIPOQ & BAFF & BDNF & CRP & EGF & MIG & THBS1 & NAP-2 \\
\hline Sex & $\begin{array}{c}0.058 \\
(\mathrm{p}=0.866)\end{array}$ & $\begin{array}{c}0.434 \\
(\mathrm{p}=0.182)\end{array}$ & $\begin{array}{c}0.115 \\
(\mathrm{p}=0.735)\end{array}$ & $\begin{array}{c}-0.059 \\
(\mathrm{p}=0.863)\end{array}$ & $\begin{array}{c}0.577 \\
(\mathrm{p}=0.063)\end{array}$ & $\begin{array}{c}-0.289 \\
(\mathrm{p}=0.389)\end{array}$ & $\begin{array}{c}0.173 \\
(p=0.611)\end{array}$ & $\begin{array}{c}-0.058 \\
(\mathrm{p}=0.865)\end{array}$ \\
\hline Age & $\begin{array}{c}0.200 \\
(\mathrm{p}=0.555)\end{array}$ & $\begin{array}{c}0.105 \\
(p=0.759)\end{array}$ & $\begin{array}{c}-0.446 \\
(\mathrm{p}=0.169)\end{array}$ & $\begin{array}{c}0.292 \\
(p=0.383)\end{array}$ & $\begin{array}{c}0.314 \\
(\mathrm{p}=0.346)\end{array}$ & $\begin{array}{c}-0.260 \\
(p=0.441)\end{array}$ & $\begin{array}{c}-0.091 \\
(\mathrm{p}=0.790)\end{array}$ & $\begin{array}{c}0.151 \\
(\mathrm{p}=0.658)\end{array}$ \\
\hline ISS stage & $\begin{array}{c}-0.443 \\
(\mathrm{p}=0.172)\end{array}$ & $\begin{array}{c}-0.321 \\
(\mathrm{p}=0.336)\end{array}$ & $\begin{array}{c}-0.183 \\
(\mathrm{p}=0.590)\end{array}$ & $\begin{array}{c}-0.185 \\
(\mathrm{p}=0.585)\end{array}$ & $\begin{array}{c}-0.255 \\
(\mathrm{p}=0.449)\end{array}$ & $\begin{array}{c}-0.087 \\
(\mathrm{p}=0.800)\end{array}$ & $\begin{array}{c}-0.005 \\
(\mathrm{p}=0.989)\end{array}$ & $\begin{array}{c}0.365 \\
(\mathrm{p}=0.293)\end{array}$ \\
\hline DS stage & $\begin{array}{c}0.000 \\
(\mathrm{p}=1.000)\end{array}$ & $\begin{array}{c}-0.672 \\
(p=0.023)\end{array}$ & $\begin{array}{c}-0.596 \\
(\mathrm{p}=0.053)\end{array}$ & $\begin{array}{c}0.459 \\
(p=0.156)\end{array}$ & $\begin{array}{c}-0.224 \\
(\mathrm{p}=0.509)\end{array}$ & $\begin{array}{c}-0.075 \\
(\mathrm{p}=0.828)\end{array}$ & $\begin{array}{c}-0.298 \\
(\mathrm{p}=0.373)\end{array}$ & $\begin{array}{c}0.487 \\
(\mathrm{p}=0.129)\end{array}$ \\
\hline Ig isotype & $\begin{array}{c}0.274 \\
(\mathrm{p}=0.476)\end{array}$ & $\begin{array}{c}0.183 \\
(\mathrm{p}=0.637)\end{array}$ & $\begin{array}{c}-0.822 \\
(p=0.007)\end{array}$ & $\begin{array}{c}-0.280 \\
(\mathrm{p}=0.466)\end{array}$ & $\begin{array}{c}-0.091 \\
(\mathrm{p}=0.815)\end{array}$ & $\begin{array}{c}0.000 \\
(\mathrm{p}=1.000)\end{array}$ & $\begin{array}{c}-0.091 \\
(\mathrm{p}=0.815)\end{array}$ & $\begin{array}{c}0.276 \\
(\mathrm{p}=0.472)\end{array}$ \\
\hline Type of light-chain & $\begin{array}{c}-0.115 \\
(\mathrm{p}=0.735)\end{array}$ & $\begin{array}{c}-0.231 \\
(\mathrm{p}=0.493)\end{array}$ & $\begin{array}{c}-0.115 \\
(\mathrm{p}=0.735)\end{array}$ & $\begin{array}{c}0.415 \\
(\mathrm{p}=0.205)\end{array}$ & $\begin{array}{c}0.000 \\
(\mathrm{p}=1.000)\end{array}$ & $\begin{array}{c}0.058 \\
(\mathrm{p}=0.866)\end{array}$ & $\begin{array}{c}-0.404 \\
(\mathrm{p}=0.218)\end{array}$ & $\begin{array}{c}-0.058 \\
(\mathrm{p}=0.865)\end{array}$ \\
\hline Hemoglobin $\left(\mathrm{g} \times \mathrm{l}^{-1}\right)$ & $\begin{array}{c}0.005 \\
(\mathrm{p}=0.989)\end{array}$ & $\begin{array}{c}0.107 \\
(p=0.753)\end{array}$ & $\begin{array}{c}-0.146 \\
(\mathrm{p}=0.669)\end{array}$ & $\begin{array}{c}0.325 \\
(p=0.330)\end{array}$ & $\begin{array}{c}-0.055 \\
(\mathrm{p}=0.873)\end{array}$ & $\begin{array}{c}0.374 \\
(\mathrm{p}=0.258)\end{array}$ & $\begin{array}{c}0.141 \\
(\mathrm{p}=0.679)\end{array}$ & $\begin{array}{c}0.064 \\
(\mathrm{p}=0.852)\end{array}$ \\
\hline Thrombocytes (amount $\times 10^{9}$ ) & $\begin{array}{c}-0.036 \\
(\mathrm{p}=0.915)\end{array}$ & $\begin{array}{c}0.383 \\
(\mathrm{p}=0.245)\end{array}$ & $\begin{array}{c}0.445 \\
(\mathrm{p}=0.170)\end{array}$ & $\begin{array}{c}-0.051 \\
(\mathrm{p}=0.881)\end{array}$ & $\begin{array}{c}0.182 \\
(p=0.593)\end{array}$ & $\begin{array}{c}-0.491 \\
(\mathrm{p}=0.125)\end{array}$ & $\begin{array}{c}0.118 \\
(p=0.729)\end{array}$ & $\begin{array}{c}-0.279 \\
(\mathrm{p}=0.407)\end{array}$ \\
\hline Calcium $\left(\mathbf{m m o l} \times \mathbf{l}^{-1}\right)$ & $\begin{array}{c}0.127 \\
(\mathrm{p}=0.709)\end{array}$ & $\begin{array}{c}-0.834 \\
(p=0.001)\end{array}$ & $\begin{array}{c}-0.036 \\
(\mathrm{p}=0.915)\end{array}$ & $\begin{array}{c}0.695 \\
(p=0.018)\end{array}$ & $\begin{array}{c}-0.336 \\
(\mathrm{p}=0.312)\end{array}$ & $\begin{array}{c}0.282 \\
(p=0.401)\end{array}$ & $\begin{array}{c}-0.500 \\
(\mathrm{p}=0.117)\end{array}$ & $\begin{array}{c}-0.046 \\
(p=0.894)\end{array}$ \\
\hline Albumin $\left(\mathrm{g} \times \mathrm{l}^{-1}\right)$ & $\begin{array}{c}-0.336 \\
(\mathrm{p}=0.312)\end{array}$ & $\begin{array}{c}0.351 \\
(\mathrm{p}=0.290)\end{array}$ & $\begin{array}{c}-0.255 \\
(\mathrm{p}=0.450)\end{array}$ & $\begin{array}{c}-0.154 \\
(\mathrm{p}=0.651)\end{array}$ & $\begin{array}{c}0.255 \\
(\mathrm{p}=0.450)\end{array}$ & $\begin{array}{c}-0.027 \\
(\mathrm{p}=0.937)\end{array}$ & $\begin{array}{c}0.627 \\
(p=0.039)\end{array}$ & $\begin{array}{c}0.489 \\
(\mathrm{p}=0.127)\end{array}$ \\
\hline Creatinine $\left(\mu \mathrm{mol} \times \mathrm{l}^{-1}\right)$ & $\begin{array}{c}-0.260 \\
(\mathrm{p}=0.441)\end{array}$ & $\begin{array}{c}-0.493 \\
(\mathrm{p}=0.123)\end{array}$ & $\begin{array}{c}-0.169 \\
(\mathrm{p}=0.620)\end{array}$ & $\begin{array}{c}0.220 \\
(\mathrm{p}=0.516)\end{array}$ & $\begin{array}{c}0.027 \\
(p=0.936)\end{array}$ & $\begin{array}{c}-0.210 \\
(\mathrm{p}=0.536)\end{array}$ & $\begin{array}{c}-0.205 \\
(p=0.545)\end{array}$ & $\begin{array}{c}0.293 \\
(\mathrm{p}=0.382)\end{array}$ \\
\hline $\mathbf{B}_{2}-$ microglobulin $\left(\mathrm{mg} \times \mathbf{l}^{-1}\right)$ & $\begin{array}{c}-0.445 \\
(\mathrm{p}=0.160)\end{array}$ & $\begin{array}{c}-0.483 \\
(\mathrm{p}=0.132)\end{array}$ & $\begin{array}{c}-0.291 \\
(\mathrm{p}=0.385)\end{array}$ & $\begin{array}{c}0.122 \\
(p=0.743)\end{array}$ & $\begin{array}{c}-0.364 \\
(\mathrm{p}=0.272)\end{array}$ & $\begin{array}{c}-0.309 \\
(p=0.355)\end{array}$ & $\begin{array}{c}-0.118 \\
(\mathrm{p}=0.729)\end{array}$ & $\begin{array}{c}0.406 \\
(p=0.215)\end{array}$ \\
\hline Lactate dehydrogenase ( $\mu$ kat) & $\begin{array}{c}0.073 \\
(\mathrm{p}=0.832)\end{array}$ & $\begin{array}{c}0.123 \\
(\mathrm{p}=0.719)\end{array}$ & $\begin{array}{c}-0.509 \\
(\mathrm{p}=0.110)\end{array}$ & $\begin{array}{c}-0.424 \\
(\mathrm{p}=0.193)\end{array}$ & $\begin{array}{c}0.027 \\
(\mathrm{p}=0.937)\end{array}$ & $\begin{array}{c}0.318 \\
(\mathrm{p}=0.340)\end{array}$ & $\begin{array}{c}0.145 \\
(p=0.670)\end{array}$ & $\begin{array}{c}0.306 \\
(\mathrm{p}=0.360)\end{array}$ \\
\hline $\mathrm{C}$-reactive protein $\left(\mathrm{mg} \times \mathrm{l}^{-1}\right)$ & $\begin{array}{c}0.336 \\
(\mathrm{p}=0.312)\end{array}$ & $\begin{array}{c}-0.442 \\
(\mathrm{p}=0.174)\end{array}$ & $\begin{array}{c}-0.327 \\
(\mathrm{p}=0.326)\end{array}$ & $\begin{array}{c}-0.023 \\
(\mathrm{p}=0.946)\end{array}$ & $\begin{array}{c}-0.691 \\
(\mathrm{p}=0.019)\end{array}$ & $\begin{array}{c}0.009 \\
(\mathrm{p}=0.979)\end{array}$ & $\begin{array}{c}-0.345 \\
(\mathrm{p}=0.298)\end{array}$ & $\begin{array}{c}0.064 \\
(\mathrm{p}=0.852)\end{array}$ \\
\hline Serum M-protein $\left(\mathrm{g} \times \mathrm{l}^{-1}\right)$ & $\begin{array}{c}0.048 \\
(\mathrm{p}=0.911)\end{array}$ & $\begin{array}{c}-0.790 \\
(\mathrm{p}=0.020)\end{array}$ & $\begin{array}{c}0.381 \\
(\mathrm{p}=0.352)\end{array}$ & $\begin{array}{c}0.195 \\
(\mathrm{p}=0.643)\end{array}$ & $\begin{array}{c}-0.643 \\
(\mathrm{p}=0.086)\end{array}$ & $\begin{array}{c}-0.143 \\
(\mathrm{p}=0.736)\end{array}$ & $\begin{array}{c}-0.476 \\
(\mathrm{p}=0.233)\end{array}$ & $\begin{array}{c}-0.325 \\
(\mathrm{p}=0.432)\end{array}$ \\
\hline BM infiltration by PCs (\%) & $\begin{array}{c}-0.006 \\
(\mathrm{p}=0.987)\end{array}$ & $\begin{array}{c}-0.596 \\
(p=0.069)\end{array}$ & $\begin{array}{c}0.445 \\
(\mathrm{p}=0.197)\end{array}$ & $\begin{array}{c}0.050 \\
(\mathrm{p}=0.890)\end{array}$ & $\begin{array}{c}-0.415 \\
(\mathrm{p}=0.233)\end{array}$ & $\begin{array}{c}0.421 \\
(p=0.226)\end{array}$ & $\begin{array}{c}-0.470 \\
(\mathrm{p}=0.171)\end{array}$ & $\begin{array}{c}-0.482 \\
(p=0.159)\end{array}$ \\
\hline
\end{tabular}

Note: significant correlations $(\mathrm{p}<0.05)$ are highlighted in bold

Abbreviations: ISS-International Staging System; DS-Durie Salmon stage; BM-bone marrow; ADIPOQ-adiponectin; BAFF-B-cell activating factor; BDNFbrain-derived neurotrophic factor; CRP-C-reactive protein; EGF-epidermal growth factor; MIG-monokine induced by gamma interferon; THBS1-thrombospondin 1; NAP-2-neutrophil activating peptide 2

expression of this protein and age, calcium, $\beta_{2}$-microglobulin, $\mathrm{LDH}$, hemoglobin, and albumin levels was observed and high levels were associated with worse overall survival of patients [38]. MIG (CXCL9), together with IP10 (CXCL10) and I-TAC (CXCL11), belongs to the CXC chemokines that bind to the CXCR3 and are associated with advanced-stage tumors, cell invasion, angiogenesis, immune escape, and disease progression. Previous studies also proved its potential to induce tyrosine-kinase phosphorylation and chemotaxis and increase the gelatinolytic activity of MMP-2 and MMP-9 [30]. These observations imply the involvement of MIG in the regulation of PCs migration capacity facilitating the formation of EMD.

Recently, Jasrotia et al. [39] measured the concentration of five selected cytokines in serum and BM compartment of newly diagnosed as well as relapsed/refractory MM patients and healthy controls. They observed deregulated levels of
BAFF in serum of MM patients compared to control samples and expression of this protein decreased significantly in BM plasma compared to the serum of newly diagnosed patients. In addition, median serum levels of BAFF were higher in relapsed/refractory patients compared to those with a new diagnosis. Similarly, two other studies detected increased expression of BAFF in the serum of MM patients compared to healthy controls [40], as well as in BM mononuclear cells and its levels correlated with the clinical stage [41]. These results are in agreement with our observations confirming the increased expression of this protein in BM plasma of EMD compared to MM patients. BAFF is known to function as an important cytokine promoting proliferation and survival of B-cells, and subsequently PCs. The binding of this protein to its receptor leads to activation of NF- $\mathrm{KB}, \mathrm{PI} 3 \mathrm{~K} /$ AKT, and MAPK signaling pathways resulting in increased proliferation, reduced apoptosis, and treatment resistance. 


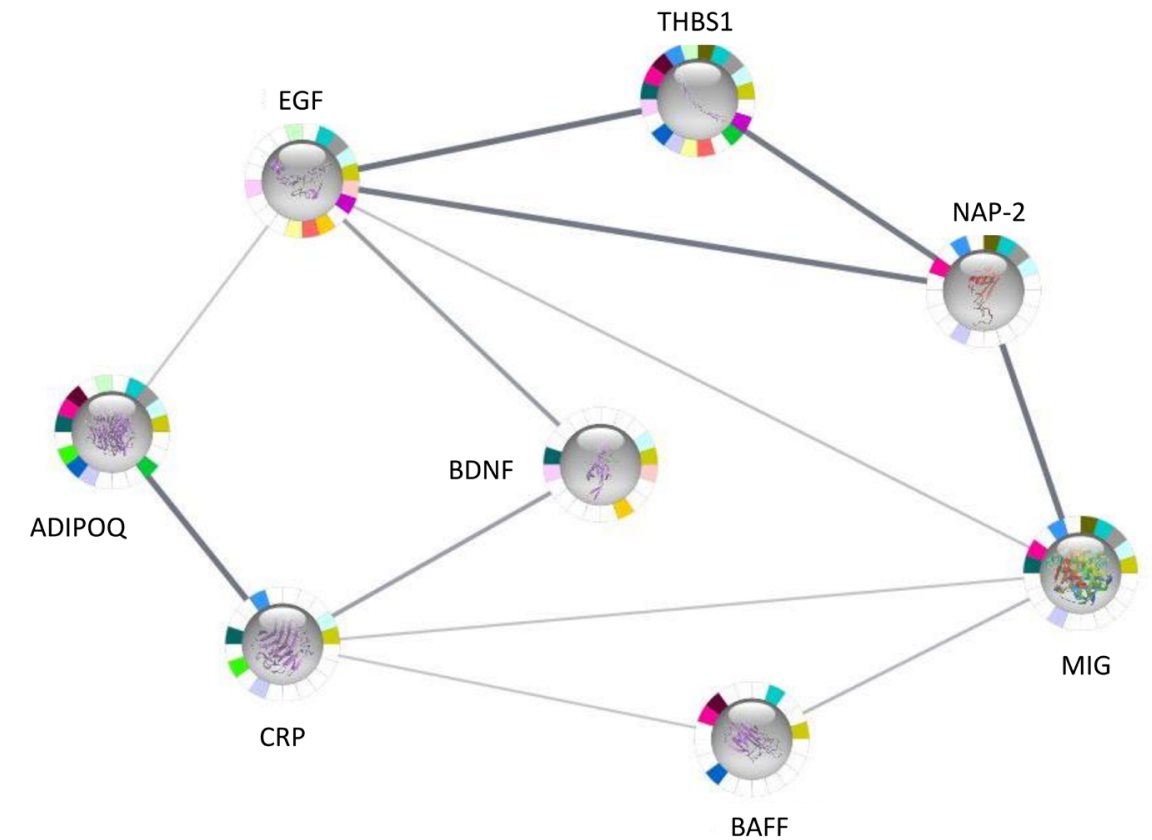

\begin{tabular}{|c|c|c|c|c|c|c|c|c|}
\hline Category & Description & Term name & Background genes & $\mathrm{N}$ & Genes & Chart color & FDR value & $P$-value \\
\hline KEGG pathway & PI3K-Akt signaling pathway & hsa04151 & 348 & 3 & THBS1, EGF, BDNF & & $5.10 \cdot 10^{-3}$ & $3.00 \cdot 10^{-4}$ \\
\hline KEGG pathway & Focal adhesion & hsa04510 & 197 & 2 & THBS1, EGF & & $2.99 \cdot 10^{-3}$ & $2.80 \cdot 10^{-3}$ \\
\hline KEGG pathway & Rap1 signaling pathway & hsa04015 & 203 & 2 & THBS1, EGF & & $2.99 \cdot 10^{-3}$ & $2.90 \cdot 10^{-3}$ \\
\hline KEGG pathway & Ras signaling pathway & hsa04014 & 228 & 2 & EGF, BDNF & & $2.99 \cdot 10^{-3}$ & $3.70 \cdot 10^{-3}$ \\
\hline KEGG pathway & MAPK signaling pathway & hsa04010 & 293 & 2 & EGF, BDNF & & $3.81 \cdot 10^{-3}$ & $6.00 \cdot 10^{-3}$ \\
\hline GO biological process & Regulation of developmental process & G0.0050793 & 2416 & 7 & CRP, THBS1, EGF, MIG, BAFF, ADIPOQ, BDNF & & $5.90 \cdot 10^{-4}$ & $3.15 \cdot 10^{-6}$ \\
\hline GO biological process & Regulation of localization & GO.0032879 & 2524 & 7 & CRP, THBS1, EGF, NAP-2, MIG, ADIPOQ, BDNF & & $6.80 \cdot 10^{-4}$ & $4.26 \cdot 10^{-6}$ \\
\hline GO biological process & Regulation of cell migration & G0.0030334 & 753 & 5 & THBS1, EGF, NAP-2, MIG, ADIPOQ & & $6.80 \cdot 10^{-4}$ & $4.37 \cdot 10^{-6}$ \\
\hline GO biological process & Positive regulation of leukocyte chemotaxis & GO.0002690 & 91 & 3 & THBS1, NAP-2, MIG & & $7.30 \cdot 10^{-4}$ & $5.90 \cdot 10^{-6}$ \\
\hline GO biological process & Regulation of cell population proliferation & GO.0042127 & 1594 & 6 & THBS1, EGF, NAP-2, MIG, BAFF, ADIPOQ & & $7.30 \cdot 10^{-4}$ & $7.17 \cdot 10^{-6}$ \\
\hline GO biological process & Regulation of lipid transport & G0.0032368 & 101 & 3 & THBS1, EGF, ADIPOQ & & $7.50 \cdot 10^{-4}$ & $8.00 \cdot 10^{-6}$ \\
\hline GO biological process & Inflammatory response & GO.0006954 & 482 & 4 & CRP, THBS1, NAP-2, MIG & & $1.00 \cdot 10^{-3}$ & $2.43 \cdot 10^{-5}$ \\
\hline GO biological process & Response to tumor necrosis factor & G0.0034612 & 217 & 3 & THBS1, BAFF, ADIPOQ & & $1.80 \cdot 10^{-3}$ & $7.52 \cdot 10^{-5}$ \\
\hline GO biological process & Regulation of immune system process & GO.0002682 & 1391 & 5 & THBS1, NAP-2, MIG, BAFF, ADIPOQ & & $2.00 \cdot 10^{-3}$ & $8.55 \cdot 10^{-5}$ \\
\hline GO biological process & Regulation of cell differentiation & GO.0045595 & 1695 & 5 & CRP, THBS1, MIG, ADIPOQ, BDNF & & $3.90 \cdot 10^{-3}$ & $2.20 \cdot 10^{-4}$ \\
\hline GO biological process & Regulation of interleukin-8 production & GO.0032677 & 67 & 2 & CRP, ADIPOQ & & $5.10 \cdot 10^{-3}$ & $3.40 \cdot 10^{4}$ \\
\hline GO biological process & Response to hypoxia & GO.0001666 & 288 & 2 & THBS1, ADIPOQ & & $3.37 \cdot 10^{-2}$ & $5.80 \cdot 10^{-3}$ \\
\hline GO biological process & Angiogenesis & GO.0001525 & 297 & 2 & THBS1, EGF & & $3.55 \cdot 10^{-2}$ & $6.10 \cdot 10^{-3}$ \\
\hline
\end{tabular}

Figure 2. Protein association network analysis. Search Tool for the Retrieval of Interacting Genes/Proteins (STRING) v 11.0 complemented with gene ontology (GO) and Kyoto Encyclopedia of Genes and Genomes (KEGG) pathways enrichment analysis was used. Abbreviations: EGF-epidermal growth factor, NAP-2-neutrophil activating peptide 2, ADIPOQ-adiponectin, CRP-C-reactive protein, BDNF-brain-derived neurotrophic factor, MIG-macrophage migration inhibitory factor, BAFF-B-cell activating factor, THBS1-thrombospondin 1, FDR-false discovery rate

Importantly, based on the results of GO and KEGG pathway enrichment analysis, most of these signaling pathways and biological processes were found to be associated with eight deregulated cytokines identified in this study. These results indicate the connection between BAFF overexpression and advanced disease and confirm the crucial role of BAFF in the pathogenesis of MM and EMD.

Based on the results of STRING analysis, a high probability of association between NAP-2 and MIG, THBS1 and EGF was found. Similar to MIG, NAP-2 is a small chemokine with a CXC motif, which origins by the cleavage of CXCL7 propeptide, and is involved in chemotaxis, degranulation of neutrophils, and megakaryocytopoiesis [42]. Previously, Matte et al. [43] observed the increased levels of NAP-2 in malignant ascites of ovarian cancer patients. Further, regulation of platelet counts by NAP-2 has been reported [44]. This is in agreement with our results showing a significant negative correlation between the expression of this chemokine and the number of thrombocytes in BM plasma of MM patients. However, this correlation was not confirmed in the 
case of EMD. THBS1 is an adhesive glycoprotein mediating the interactions with other cells and ECM of the BM microenvironment through binding to different adhesion receptors including $\mathrm{CD} 36$, syndecan, $\mathrm{CD} 47$, or $\beta 1$ integrins. It plays an essential role in platelet aggregation, angiogenesis, cell motility, and tumor growth, which corresponds with the increased expression in EMD compared to MM. Elevated levels of THBS1 together with its receptor CD47 were detected in PCs of MM patients compared to MGUS and healthy controls [45]. Interestingly, disruption of CD47-THBS-1 interaction by blocking antibodies or RNA interference abrogated the tumor-induced osteoclast formation, attenuated hypercalcemia, and inhibited RANKL and macrophage colony-stimulating factor (M-CSF)-induced formation of osteoclasts from human monocytes, indicating the dominant role for THBS-1-CD47 interactions in myeloma-induced differentiation of dendritic cells into osteoclasts [46]. Interestingly, high pretreatment levels of THBS1 in BM plasma of MM patients were associated with complete or very good partial response to high-dose chemotherapy by bortezomib $[10,47]$.

Protein association network analysis also revealed the strong association between ADIPOQ and CRP as both these proteins are involved in the regulation of cell development, localization, differentiation, response to stress, and production of IL-8. Our results did not prove any correlation between the expressions of these two cytokines in BM plasma of MM/ EMD patients. However, a negative correlation between the BM plasma levels of ADIPOQ and blood serum levels of CRP was confirmed. ADIPOQ is a protein hormone secreted into the bloodstream mainly by adipocyte cells to decrease cell proliferation, and its levels are inversely proportional to the amount of adipose tissue. Thus, an excessive amount of adipose tissue is considered a risk factor for the development of MM [48]. Earlier studies found decreased levels of ADIPOQ in the serum of MGUS patients, who subsequently progressed to MM compared to those with no progression [49]. At the same time, increased expression of this protein was detected in serum samples of MGUS patients compared to smoldering MM or fully developed MM. In addition, low levels of ADIPOQ were associated with the higher-risk IgM isotype $[50,51]$. In the recent study, inhibition of the differentiation and maturation of osteoclasts by ADIPOQ by increasing the expression of ADIPOR1 and reducing the phosphorylation levels of mTOR in patients with MM was described [52]. Importantly, Morris et al. [53] demonstrated that myeloma cells are able to downregulate ADIPOQ in BM adipocytes via the TNF- $\alpha$ signaling pathway that leads to the alteration of BM microenvironment and disease progression indicating the possible mechanism of EMD development.

CRP is an acute-phase protein of hepatic origin that is synthesized in response to factors released by macrophages, T-cells, and adipocytes, especially IL-6. Previously, decreased CRP levels were observed in the serum of MGUS patients compared to MM patients at the time of diagnosis, and overexpression of this protein was associated with shorter overall survival [54]. Further, serum levels of CRP together with IL-6 were found to be significantly upregulated in MM patients compared to healthy controls, and expression of these cytokines decreased after vincristine-adriamycindexamethasone-based chemotherapy [55]. In addition, elevated levels of pre-transplant CRP enabled to identify a high-risk subgroup of MM patients undergoing delayed autologous stem cell transplantation [56]. In our study, BM plasma levels of CRP were significantly reduced in MM patients compared to EMD patients; this change corresponded to the difference in serum CRP levels. Further, a positive correlation between the levels of this protein and BMPCs infiltration was observed. As decreased immunity associated with recurrent and complicated infections is commonly present in MM patients [57], disease progression may be reflected in the worsening of the clinical condition, increased rate of infections, and consequently elevated expression of CRP. In vitro and in vivo studies revealed that CRP enhances myeloma cells' proliferation under stressed conditions through activating PI3K/AKT, ERK, and NF- $\kappa B$ signaling pathways, increases the secretion of IL-6, and protects the cells from chemotherapy-induced apoptosis due to the inhibition of caspase cascade [58]. Besides that, CRP activates the p38 MAPK-TWIST pathway and enhances the secretion of osteolytic cytokines by myeloma cells promoting osteoclastogenesis and bone destruction [59].

Finally, an increased expression of BDNF in BM plasma of MM patients was detected compared to EMD patients and negatively correlated with ISS stage and $\beta_{2}$-microglobulin levels. These results seem to be contradictory to previous papers reporting the oncogenic function of this growth factor with increased expression in advanced disease [60-63]. Nevertheless, none of these studies measured the levels of BDNF in EMD patients. In MM patients, BDNF is expressed primarily in the BM by stromal cells, osteoblasts, and endothelial cells, indicating the critical role of this protein in the interaction of MM cells with bone and stroma [60]. Further studies reported that binding of BDNF to tyrosine receptor kinase B (TrkB) activates the PI3K/AKT and MAPK signaling pathways, which leads to increased proliferation, migration, and survival of myeloma cells [62] as well as enhanced angiogenesis due to the VEGF upregulation [63]. In addition, disruption of the balance between the RANKL and osteoprotegerin expression was observed in MM patients with deregulated levels of BDNF and associated with increased osteoclastogenesis [64]. Recently, serum concentration of BDNF was correlated with the severity of polyneuropathy and response to chemotherapy. Interestingly, low levels of this protein were found in MM patients with worse overall survival [65] indicating a possible presence of EMD.

In conclusion, MM is a very heterogeneous hematological malignancy with a poor prognosis, which is mostly associated with the presence of aggressive EMD. Recently, different mechanisms responsible for the colonization of 
BM niches by MM cells and their escape during the extramedullary stages of the disease have been clarified and the key role of several soluble molecules in the pathogenesis of MM was described. In this study, eight different cytokines with a significantly deregulated expression between the BM plasma samples of MM patients and patients with EMD have been identified and correlated with selected clinical-pathological features of patients. Based on the results of GO and KEGG pathways enrichment analysis, engagement of these cytokines in the regulation of cell proliferation, migration, differentiation, adhesion, and immune response was confirmed and linked to the activation of the PI3K/AKT and MAPK signaling pathways. These results indicate the complex involvement of numerous cytokines in the pathogenesis of MM and the crucial role of BM microenvironment in the disease progression. Thus, a deeper understanding of these processes may contribute to the development and clinical application of more effective targeted therapies inhibiting the progression of MM into EMD, and thus improving the patient's survival.

Acknowledgments: The authors would like to thank all patients participating in this study and their caregivers. This work was supported by grant AZV 17-29343A.

\section{References}

[1] SIEGEL RL, MILLER KD, JEMAL A. Cancer statistics, 2019. CA Cancer J Clin 2019; 69: 7-34. https://doi.org/10.3322/ caac. 21551

[2] RAJKUMAR SV. Multiple myeloma: 2020 update on diagnosis, risk-stratification and management. Am J Hematol 2020; 95: 548-567. https://doi.org/10.1002/ajh.25791

[3] DAVIS LN, SHERBENOU DW. Emerging Therapeutic Strategies to Overcome Drug Resistance in Multiple Myeloma. Cancers (Basel) 2021; 13. https://doi.org/10.3390/cancers 13071686

[4] JELINEK T, KORISTKA M, CERMAKOVA Z, HAJEK R. Daratumumab - Hope for Myeloma Patients, a Challenge for Clinical Laboratories. Klin Onkol 2017; 30: 13-19. https:// doi.org/10.14735/amko201713.

[5] KUBICZKOVA L, MATEJIKOVA J, SEDLARIKOVA L, KRYUKOV F, HAJEK R et al. Proteasome inhibitors in treatment of multiple myeloma. Klin Onkol 2013; 26: 11-18. https://doi.org/10.14735/amko201311

[6] ROZIAKOVA L, MISTRIK M, BATOROVA A. Pomalidomide in the treatment of relapsed and refractory multiple myeloma. Klin Onkol 2014; 27: 318-325. https://doi. org/10.14735/amko2014318

[7] SEVCIKOVA S, PASZEKOVA H, BESSE L, SEDLARIKOVA L, KUBACZKOVA V et al. Extramedullary relapse of multiple myeloma defined as the highest risk group based on deregulated gene expression data. Biomed Pap Med Fac Univ Palacky Olomouc Czech Repub 2015; 159: 288-293. https:// doi.org/10.5507/bp.2015.014
[8] SEVCIKOVA S, MINARIK J, STORK M, JELINEK T, POUR L et al. Extramedullary disease in multiple myeloma - controversies and future directions. Blood Rev 2019; 36: 32-39. https://doi.org/10.1016/j.blre.2019.04.002

[9] WEINSTOCK M, GHOBRIAL IM. Extramedullary multiple myeloma. Leuk Lymphoma 2013; 54: 1135-1141. https://doi. org/10.3109/10428194.2012.740562

[10] POUR L, SEVCIKOVA S, GRESLIKOVA H, KUPSKA R, MAJKOVA $\mathrm{P}$ et al. Soft-tissue extramedullary multiple myeloma prognosis is significantly worse in comparison to bone-related extramedullary relapse. Haematologica 2014; 99: 360-364. https://doi.org/10.3324/haematol.2013.094409.

[11] BATSUKH K, LEE SE, MIN GJ, PARK SS, JEON YW et al. Distinct Clinical Outcomes between Paramedullary and Extramedullary Lesions in Newly Diagnosed Multiple Myeloma. Immune Netw 2017; 17: 250-260. https://doi. org/10.4110/in.2017.17.4.250

[12] FERNANDEZ de LARREA C, KYLE RA, DURIE BGM, LUDWIG H, USMANI S et al. Plasma cell leukemia: consensus statement on diagnostic requirements, response criteria and treatment recommendations by the International $\mathrm{My}$ eloma Working Group. Leukemia 2013; 27: 780-291. https:// doi.org/10.1038/leu.2012.336

[13] PAGET S. The distribution of secondary growths in cancer of the breast. 1889. Cancer Metastasis Rev 1989; 8: 98-101.

[14] LANGLEY RR, FIDLER IJ. The seed and soil hypothesis revisited--the role of tumor-stroma interactions in metastasis to different organs. Int J Cancer 2011; 128: 2527-2535. https://doi.org/10.1002/ijc.26031

[15] WEI Q, FRENETTE PS. Niches for Hematopoietic Stem Cells and Their Progeny. Immunity 2018; 48: 632-648. https://doi.org/10.1016/j.immuni.2018.03.024

[16] GARCIA-ORTIZ A, RODRIGUEZ-GARCIA Y, ENCINAS J, MAROTO-MARTIN E, CASTELLANO E et al. The Role of Tumor Microenvironment in Multiple Myeloma Development and Progression. Cancers (Basel) 2021; 13. https://doi. org/10.3390/cancers 13020217

[17] ULLAH TR. The role of CXCR4 in multiple myeloma: Cells' journey from bone marrow to beyond. J Bone Oncol 2019; 17. https://doi.org/10.1016/j.jbo.2019.100253

[18] MARTINEZ-MORENO M, LEIVA M, AGUILERA-MONTILLAN, SEVILLA-MOVILLAS, ISERN de VALS etal.Invivo adhesion of malignant $B$ cells to bone marrow microvasculature is regulated by $\alpha 4 \beta 1$ cytoplasmic-binding proteins. Leukemia 2016; 30: 861-872. https://doi.org/10.1038/leu.2015.332

[19] TARNOWSKI M, GRYMULA K, LIU R, TARNOWSKA J, DRUKALA J et al. Macrophage migration inhibitory factor is secreted by rhabdomyosarcoma cells, modulates tumor metastasis by binding to CXCR4 and CXCR7 receptors and inhibits recruitment of cancer-associated fibroblasts. Mol Cancer Res 2010; 8: 1328-1343. https://doi. org/10.1158/1541-7786.MCR-10-0288

[20] STESSMAN H a. F, MANSOOR A, ZHAN F, JANZ S, LINDEN MA et al. Reduced CXCR4 expression is associated with extramedullary disease in a mouse model of myeloma and predicts poor survival in multiple myeloma patients treated with bortezomib. Leukemia 2013; 27: 2075-2077. https://doi.org/10.1038/leu.2013.148 
[21] GOODING S, EDWARDS CM. New approaches to targeting the bone marrow microenvironment in multiple myeloma. Curr Opin Pharmacol 2016; 28: 43-49. https://doi. org/10.1016/j.coph.2016.02.013

[22] GREGOROVA J, VRABEL D, RADOVA L, GABLO NA, ALAMSI $\mathrm{M}$ et al. MicroRNA Analysis for Extramedullary Multiple Myeloma Relapse. Klin Onkol 2018; 3 1:148-150.

[23] GIANNAKOULAS N, NTANASIS-STATHOPOULOS I, TERPOS E. The Role of Marrow Microenvironment in the Growth and Development of Malignant Plasma Cells in Multiple Myeloma. Int J Mol Sci 2021; 22: 4462. https://doi. org/10.3390/ijms22094462

[24] MITSIADES CS, MITSIADES NS, MUNSHI NC, RICHARDSON PG, ANDERSON KC. The role of the bone microenvironment in the pathophysiology and therapeutic management of multiple myeloma: Interplay of growth factors, their receptors and stromal interactions. Eur J Cancer 2006; 42: 1564-1573. https://doi.org/10.1016/j.ejca.2005.12.025

[25] GIULIANI N, BONOMINI S, ROMAGNANI P, LAZZARETTI M, MORANDI $F$ et al. CXCR3 and its binding chemokines in myeloma cells: expression of isoforms and potential relationships with myeloma cell proliferation and survival. Haematologica 2006; 91: 1489-1497.

[26] MÖLLER C, STRÖMBERG T, JUREMALM M, NILSSON K, NILSSON G. Expression and function of chemokine receptors in human multiple myeloma. Leukemia 2003; 17: 203-210. https://doi.org/10.1038/sj.leu.2402717

[27] BROEK IV, ASOSINGH K, VANDERKERKEN K, STRAETMANS N, VAN CAMP B et al. Chemokine receptor CCR2 is expressed by human multiple myeloma cells and mediates migration to bone marrow stromal cell-produced monocyte chemotactic proteins MCP-1, -2 and -3. Br J Cancer 2003; 88: 855-862. https://doi.org/10.1038/sj.bjc.6600833

[28] MENU E, DE LEENHEER E, DE RAEVE H, COULTON L, IMANISHI T et al. Role of CCR1 and CCR5 in homing and growth of multiple myeloma and in the development of osteolytic lesions: a study in the 5TMM model. Clin Exp Metastasis 2006; 23: 291-300. https://doi.org/10.1007/s10585006-9038-6

[29] ALSAYED Y, NGO H, RUNNELS J, LELEU X, SINGHA UK et al. Mechanisms of regulation of CXCR4/SDF-1 (CXCL12)dependent migration and homing in multiple myeloma. Blood 2007; 109: 2708-2717. https://doi.org/10.1182/ blood-2006-07-035857

[30] PELLEGRINO A, ANTONACI F, RUSSO F, MERCHIONNE F, RIBATTI D et al. CXCR3-binding chemokines in multiple myeloma. Cancer Lett 2004; 207: 221-227. https://doi. org/10.1016/j.canlet.2003.10.036

[31] MUSOLINO C, ALLEGRA A, INNAO V, ALLEGRA AG, PIOGGIA $G$ et al. Inflammatory and Anti-Inflammatory Equilibrium, Proliferative and Antiproliferative Balance: The Role of Cytokines in Multiple Myeloma. Mediators Inflamm 2017; 2017: 1852517. https://doi.org/10.1155/2017/1852517

[32] BERGMANN R, LUDBROOK J, SPOOREN WPJM. Different Outcomes of the Wilcoxon-Mann-Whitney Test from Different Statistics Packages. The American Statistician 2000; 54: 72-77. https://doi.org/10.1080/00031305.2000.10474513
[33] SHANNON P, MARKIEL A, OZIER O, BALIGA NS, WANG JT et al. Cytoscape: a software environment for integrated models of biomolecular interaction networks. Genome Res 2003; 13: 2498-2504. https://doi.org/10.1101/gr.1239303

[34] SZKLARCZYK D, GABLE AL, NASTOU KC, LYON D, KIRSCH R et al. The STRING database in 2021: customizable protein-protein networks, and functional characterization of user-uploaded gene/measurement sets. Nucleic Acids Res 2021; 49: D605-612. https://doi.org/10.1093/nar/ gkaa1074

[35] BHUTANI M, FOUREAU DM, ATRASH S, VOORHEES PM, USMANI SZ. Extramedullary multiple myeloma. Leukemia 2020; 34: 1-20. https://doi.org/10.1038/s41375-0190660-0

[36] CAO Y, LUETKENS T, KOBOLD S, HILDEBRANDT Y, GORDIC $\mathrm{M}$ et al. The cytokine/chemokine pattern in the bone marrow environment of multiple myeloma patients. Exp Hematol 2010; 38: 860-8677. https://doi.org/10.1016/j. exphem.2010.06.012

[37] ROBAK P, WEGLOWSKA E, DROZDZ I, MIKULSKI D, JARYCH D et al. Cytokine and Chemokine Profile in Patients with Multiple Myeloma Treated with Bortezomib. Mediators Inflamm 2020; 2020. https://doi.org/10.1155/2020/1835836

[38] BOLOMSKY A, ZOJER N, SCHREDER M, LUDWIG H. CXCR3 Binding Chemokines MIG, IP-10 and ITAC Are Predictors of Overall Survival in Newly Diagnosed Multiple Myeloma. Blood 2014; 124: 2052. https://doi.org/10.1182/ blood.V124.21.2052.2052

[39] JASROTIA S, GUPTA R, SHARMA A, HALDER A, KUMAR L. Cytokine profile in multiple myeloma. Cytokine 2020; 136: 155271. https://doi.org/10.1016/j.cyto.2020.155271

[40] MOREAUX J, LEGOUFFE E, JOURDAN E, QUITTET $\mathrm{P}, \mathrm{REME} \mathrm{T}$ et al. BAFF and APRIL protect myeloma cells from apoptosis induced by interleukin 6 deprivation and dexamethasone. Blood 2004; 103: 3148-3157. https://doi. org/10.1182/blood-2003-06-1984

[41] PAN J, SUN Y, ZHANG N, LI J, TA F et al. Characteristics of BAFF and APRIL factor expression in multiple myeloma and clinical significance. Oncol Lett 2017; 14: 2657-2662. https:// doi.org/10.3892/ol.2017.6528

[42] PILLAI MM, IWATA M, AWAYA N, GRAF L, TOROKSTORB B. Monocyte-derived CXCL7 peptides in the marrow microenvironment. Blood 2006; 107: 3520-3526. https://doi.org/10.1182/blood-2005-10-4285

[43] MATTE I, LANE D, LAPLANTE C, RANCOURT C, PICHE A. Profiling of cytokines in human epithelial ovarian cancer ascites. Am J Cancer Res 2012; 2: 566-580.

[44] ODA M, KURASAWA Y, TODOKORO K, NAGATA Y. Thrombopoietin-induced CXC chemokines, NAP-2 and PF4, suppress polyploidization and proplatelet formation during megakaryocyte maturation. Genes Cells 2003; 8: 9-15. https://doi.org/10.1046/j.1365-2443.2003.00610.x

[45] DANIELSEN JMR, KNUDSEN LM, DAHL IM, LODAHL M, RASMUSSEN T. Dysregulation of CD47 and the ligands thrombospondin 1 and 2 in multiple myeloma. Br J Haematol 2007; 138: 756-760. https://doi.org/10.1111/j.13652141.2007.06729.x 
[46] KUKREJA A, RADFAR S, SUN BH, INSOGNA K, DHODAPKAR MV. Dominant role of CD47-thrombospondin-1 interactions in myeloma-induced fusion of human dendritic cells: implications for bone disease. Blood 2009; 114: 34133421. https://doi.org/10.1182/blood-2009-03-211920

[47] POUR L, SVACHOVA H, ADAM Z, MIKULKOVA Z, BURESOVA L et al. Pretreatment hepatocyte growth factor and thrombospondin-1 levels predict response to high-dose chemotherapy for multiple myeloma. Neoplasma 2010; 57: 29-34. https://doi.org/10.4149/neo_2010_01_029

[48] HOFMANN JN, BIRMANN BM, TERAS LR, PFEIFFER RM, WANG Y et al. Low Levels of Circulating Adiponectin Are Associated with Multiple Myeloma Risk in Overweight and Obese Individuals. Cancer Res 2016; 76: 1935-1941. https://doi.org/10.1158/0008-5472.CAN-15-2406

[49] FOWLER JA, LWIN ST, DRAKE MT, EDWARDS JR, KYLE RA et al. Host-derived adiponectin is tumor-suppressive and a novel therapeutic target for multiple myeloma and the associated bone disease. Blood 2011; 118: 5872-5882. https:// doi.org/10.1182/blood-2011-01-330407

[50] HOFMANN JN, MAILANKODY S, KORDE N, WANG Y, TAGEJA N et al. Circulating Adiponectin Levels Differ Between Patients with Multiple Myeloma and its Precursor Disease. Obesity (Silver Spring) 2017; 25: 1317-1320. https:// doi.org/10.1002/oby.21894

[51] DALAMAGA M, KARMANIOLAS K, PANAGIOTOU A, HSI A, CHAMBERLAND J et al. Low circulating adiponectin and resistin, but not leptin, levels are associated with multiple myeloma risk: a case-control study. Cancer Causes Control 2009; 20: 193-199. https://doi.org/10.1007/s10552008-9233-7

[52] LIU Z, LIU H, LI Y, WANG Y, XING R et al. Adiponectin inhibits the differentiation and maturation of osteoclasts via the mTOR pathway in multiple myeloma. Int J Mol Med 2020; 45: 1112-1120. https://doi.org/10.3892/ijmm.2020.4475

[53] MORRIS EV, SUCHACKI KJ, HOCKING J, CARTWRIGHT R, SOWMAN A et al. Myeloma Cells Down-Regulate Adiponectin in Bone Marrow Adipocytes Via TNF-Alpha. J Bone Miner Res 2020; 35: 942-955. https://doi.org/10.1002/ jbmr.3951

[54] BATAILLE R, BOCCADORO M, KLEIN B, DURIE B, PILERI A. C-Reactive Protein and 0-2 Microglobulin Produce a Simple and Powerful Myeloma Staging System. Blood 1992; 80: 733-737. https://doi.org/10.1182/blood.V80.3.733.733

[55] KUKU I, BAYRAKTER MR, KAYA E, ERKURT MA, BAYRAKTAR $\mathrm{N}$ et al. Serum proinflammatory mediators at different periods of therapy in patients with multiple myeloma. Mediators Inflamm 2005; 2005: 171-174. https://doi. org/10.1155/MI.2005.171
[56] CHAKRABORTY R, MUCHTAR E, KUMAR SK, BUADI FK, DINGLI D et al. Elevated pre-transplant C-reactive protein identifies a high-risk subgroup in multiple myeloma patients undergoing delayed autologous stem cell transplantation. Bone Marrow Transplant 2018; 53: 155-161. https:// doi.org/10.1038/bmt.2017.228

[57] HAJEK R, KREJCI M, POUR L, ADAM Z. Multiple myeloma. Klin Onkol 2011; 24: S10-13.

[58] YANG J, WEZEMAN M, ZHANG X, LIN P, WANG M et al. Human C-Reactive Protein Binds Activating Fc $\gamma$ Receptors and Protects Myeloma Tumor Cells from Apoptosis. Cancer Cell 2007; 12: 252-265. https://doi.org/10.1016/j. ccr.2007.08.008

[59] YANG J, LIU Z, LIU H, HE J, YANG J et al. C-reactive protein promotes bone destruction in human myeloma through the CD32-p38MAPK-Twist axis. Sci Signal 2017; 10. https:// doi.org/10.1126/scisignal.aan6282

[60] PEARSE RN, SWENDEMAN SL, LI Y, RAFII D, HEMPSTEAD BL. A neurotrophin axis in myeloma: TrkB and BDNF promote tumor-cell survival. Blood 2005; 105: 4429_ 4436. https://doi.org/10.1182/blood-2004-08-3096

[61] SUN C, HU Y, HUANG J, CHU Z, ZHANG L et al. Brainderived neurotrophic factor induces proliferation, migration, and VEGF secretion in human multiple myeloma cells via activation of MEK-ERK and PI3K/AKT signaling. Tumor Biol 2010; 31: 121-128. https://doi.org/10.1007/s13277-0100016-X

[62] HU Y, SUN C, WANG H, GUO T, WEI W et al. Brain-derived neurotrophic factor promotes growth and migration of multiple myeloma cells. Cancer Genet Cytogenet 2006; 169: 12-20. https://doi.org/10.1016/j.cancergencyto.2006.02.018

[63] HU Y, WANG Y, GUO T, WEI W, SUN C et al. Identification of brain-derived neurotrophic factor as a novel angiogenic protein in multiple myeloma. Cancer Genet Cytogenet 2007; 178: 1-10. https://doi.org/10.1016/j.cancergencyto.2007.05.028

[64] AI LS, SUN CY, WANG YD, ZHANG L, CHU ZB et al. Gene silencing of the BDNF/TrkB axis in multiple myeloma blocks bone destruction and tumor burden in vitro and in vivo. Int J Cancer 2013; 133: 1074-1084. https://doi.org/10.1002/ ijc. 28116

[65] SZUDY-SZCZYREK A, MLAK R, BURY-KAMINSKA M, MIELNIK M, PODGAJNA $M$ et al. Serum brain-derived neurotrophic factor (BDNF) concentration predicts polyneuropathy and overall survival in multiple myeloma patients. Br J Haematol 2020; 191: 77-89. https://doi.org/10.1111/ bjh. 16862 